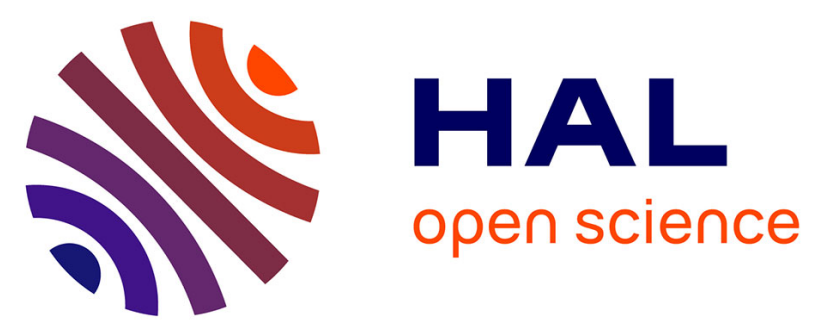

\title{
(SI) Reorganization free energies and quantum corrections for a model electron self-exchange reaction: Comparison of polarizable and nonpolarizable solvent models
}

Jochen Blumberger, Guillaume Lamoureux

\section{To cite this version:}

Jochen Blumberger, Guillaume Lamoureux. (SI) Reorganization free energies and quantum corrections for a model electron self-exchange reaction: Comparison of polarizable and nonpolarizable solvent models. Molecular Physics, 2008, 106 (12-13), pp.1597-1611. 10.1080/00268970802220112 . hal00513212

\section{HAL Id: hal-00513212 \\ https://hal.science/hal-00513212}

Submitted on 1 Sep 2010

HAL is a multi-disciplinary open access archive for the deposit and dissemination of scientific research documents, whether they are published or not. The documents may come from teaching and research institutions in France or abroad, or from public or private research centers.
L'archive ouverte pluridisciplinaire HAL, est destinée au dépôt et à la diffusion de documents scientifiques de niveau recherche, publiés ou non, émanant des établissements d'enseignement et de recherche français ou étrangers, des laboratoires publics ou privés. 


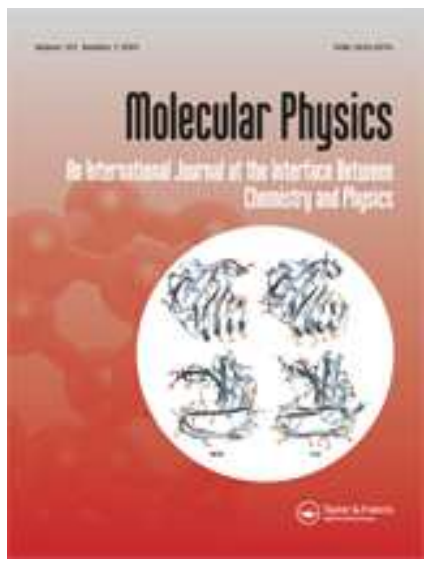

(SI) Reorganization free energies and quantum corrections for a model electron self-exchange reaction: Comparison of polarizable and nonpolarizable solvent models

\begin{tabular}{|r|l|}
\hline Journal: & Molecular Physics \\
\hline Manuscript ID: & TMPH-2008-0101.R1 \\
\hline Manuscript Type: & Invited Article \\
\hline Author: & 17-May-2008 \\
\hline Complete List of Authors: & $\begin{array}{l}\text { Blumberger, Jochen; Cambridge University, Chemistry } \\
\text { Lamoureux, Guillaume; Concordia University, Chemistry and } \\
\text { Biochemistry }\end{array}$ \\
\hline Keywords: & $\begin{array}{l}\text { reorganization energy, electron transfer, polarizable water models, } \\
\text { molecular dynamics, quantum correction }\end{array}$ \\
\hline & \\
\hline $\begin{array}{l}\text { Note: The following files were submitted by the author for peer review, but cannot be converted } \\
\text { to PDF. You must view these files (e.g. movies) online. }\end{array}$ \\
\hline ru.tex
\end{tabular}

\section{scholarONE \\ Manuscript Central}




\title{
Reorganization free energies and quantum corrections for a model electron
} self-exchange reaction: Comparison of polarizable and nonpolarizable solvent models

\author{
Jochen Blumberger*, ${ }^{\dagger}$, Guillaume Lamoureux ${ }^{+}$ \\ May 17, 2008 \\ Department of Chemistry, University of Cambridge, Lensfield Road, Cambridge CB2 1EW, UK. \\ Department of Chemistry and Biochemistry, Concordia University, 7141 rue Sherbrooke Ouest, Montréal, Québec H4B \\ 1R6, Canada. \\ *University of Cambridge, e-mail: jb376@cam.ac.uk, phone: $++44-(0) 1223-763872$, fax: $++44-(0) 1223-336362$ \\ +Concordia University, e-mail: guillaume.lamoureux@concordia.ca, phone: (+1)514-848-2424 x5314, fax: \\ $(+1) 514-848-2868$ \\ $\dagger$ corresponding author
}

\begin{abstract}
The solvent contribution to the reorganization free energy for electron self-exchange in aqueous $\mathrm{Ru}(\mathrm{II})$ $\mathrm{Ru}(\mathrm{III})$ is computed for two recently developed polarizable water models, AMOEBA [Ren, P.; Ponder, J. W. J. Phys. Chem. B, 2003, 10\%, 5933] and SWM4-NDP [Lamoureux, G.; Harder, E.; Vorobyov, I. V.; Roux, B.; MacKerell Jr, A. D. Chem. Phys. Lett., 2005, 418, 241], and for the earlier POL3 model [Caldwell, J. W.; Kollman, P. A. J. Phys. Chem., 1995, 99, 6208], and compared to the reorganization free energy of nonpolarizable water models. The "solute", defined as the two ions and their first hydration shells, is treated as nonpolarizable. We find that the solvent ("outer sphere") reorganization free energy is reduced by $22 \%$ for SWM4-NDP and by $11 \%$ for POL3 relative to the nonpolarizable TIP3P water, but increased by $5 \%$ for AMOEBA water. This is less than the $\approx 38 \%$ reduction suggested by standard continuum theory and confirms the view that continuum theory predicts a stronger dependence of solvent reorganization on the optical dielectric constant than what is obtained from atomistic simulation. The varying degree of reduction in reorganization free energy for polarizable water models is the consequence of two opposing effects: (i) reduction in reorganization free energy due to decreased electronic response, and (ii) increase in reorganization free energy due to increased nuclear response. The first effect gives a consistent decrease of about $30 \%$ while the second effect strongly depends on the polarizable water model used and is largest for AMOEBA water. Rate enhancements due to quantum corrections are computed in the harmonic bath approximation and range between 3.8-10.9 in good agreement with the estimate obtained from experimental dispersion data of liquid water, 7.7. The rigid nonpolarizable water models overestimate the quantum correction in the libration modes which effectively compensates for the neglect of quantum corrections in the absent stretching modes. About $85 \%$ of the solvent reorganization is due to the second and third solvation shell of the ion pair. Size effects caused by the finite number of solvent molecules are minor and much smaller than for oxidation of a single ion.
\end{abstract}




\section{Introduction}

In Marcus theory of electron transfer $(\mathrm{ET}),{ }^{1-5}$ the activation free energy is determined by two essential quantities: the driving force and the reorganization free energy. Formally, the reorganization free energy is equal to the free energy required to change ("reorganize") the solute and solvent from the equilibrium configuration of the reactant to the equilibrium configuration of the product while staying on the same diabatic surface. Describing the solvent as a continuum, Marcus has provided a formula for the solvent contribution to reorganization. It is predicted to be proportional to the charge transferred, $\Delta q$, to the difference of the inverse radii $r_{1}, r_{2}$ of the spherical solute cavities and the ion-ion separation $R$, and to the Pekar factor of the solvent, $\epsilon_{\mathrm{op}}^{-1}-\epsilon_{\mathrm{s}}^{-1}$, where $\epsilon_{\mathrm{op}}$ and $\epsilon_{\mathrm{s}}$ are the optical and static dielectric constants. ${ }^{1}$

$$
\lambda_{\mathrm{o}}^{\mathrm{cl}}=(\Delta q)^{2}\left(\frac{1}{\epsilon_{\mathrm{op}}}-\frac{1}{\epsilon_{\mathrm{s}}}\right)\left(\frac{1}{2 r_{1}}+\frac{1}{2 r_{2}}-\frac{1}{R}\right)
$$

Equation 1 is one of the cornerstones of Marcus theory, allowing to estimate reorganization just from the dielectric properties of the solvent and the size of the ions. (The superscript "cl" refers to the classical bath limit.) A drawback of this model and of most continuum models in general is the strong dependence of the energetics on the choice of the cavity radii. There is no unique definition of the radii and it is often difficult to find a single set of radii that adequately describe all available data such as ionic solvation free energies and reorganization free energies. ${ }^{6,7}$ By construction Eq. 1 seems less appropriate for estimation of reorganization of highly heterogeneous systems such as proteins. In such a case $\lambda_{0}$ has to be estimated with other methods and then used as an input in Eq. 1 to calculate an effective dielectric constant of the medium. Despite the many successful applications of Eq. 1 its predictive power is somewhat limited, begging the question: Can solvent ("outer sphere") reorganization be calculated with more refined methods that take into account all or most of the microscopic detail?

Indeed, since the pioneering work of Warshel ${ }^{8}$ much effort has been invested to estimate reorganization free energies on a microscopic level using molecular simulation. ${ }^{6,7,9-30}$ Model systems typically comprised of a donoracceptor pair surrounded by a few hundred rigid and nonpolarizable solvent molecules. For this setup the reorganization free energy was found to be systematically overestimated ${ }^{9,10,12,14,20,29}$ when compared to estimates based on experimental data. A possible reason for this is easily found when one considers Marcus' continuum expression Eq. 1. The standard solvent models lack electronic polarization $\left(\epsilon_{\mathrm{op}}=1\right)$ and if $\epsilon_{\mathrm{s}}>>1$ the reorganization is overestimated by a factor equal to the experimental optical dielectric constant, $\epsilon_{\mathrm{op}}=1.5-2.5$ for most polar media and 1.78 for water. This explains very well the large solvent reorganization of $2.34 \mathrm{eV}$ for ferrous-ferric self-exchange ${ }^{9}$ (in nonpolarizable SPC water) when compared to the value taken to fit experimental data, $1.20 \mathrm{eV} .{ }^{31}$ Since then the problem of missing electron polarizability has often been circumvented by scaling ("renormalizing") the reorganization free energies obtained with nonpolarizable solvent models by the ratio of Pekar factors of experimental and simulated water. ${ }^{12,14}$

A more rigorous approach to this problem is the inclusion of explicit electronic polarization in the molecular dynamics simulation. The results of such electron transfer (ET) simulations have been controversial so far. Polarizable models based on fluctuating atomic charges ${ }^{6,13,15,32}$ give a decrease in solvent reorganization of only $10 \%$ relative to the corresponding nonpolarizable models. This is much less than the $\approx 45 \%$ reduction implied by the ratio of Pekar factors. A more significant reduction of reorganization was reported when polarization was included through polarizable atomic dipoles. Warshel and co-workers, for instance, reported a decrease of about $34 \%$ for a model ET reaction between $\mathrm{Na}^{+}$and $\mathrm{Cl}^{-} .{ }^{10}$ Marchi and co-workers reported an even larger decrease of $36-50 \%$ for ET in the bacterial reaction center. ${ }^{20}$

Another controversial issue is the importance of nuclear quantum effects of the solvent. Chandler and cowork$e^{11}$ reported a tunneling enhancement of 65 for ferrous-ferric self-exchange from path integral Monte Carlo simulation. Under a harmonic approximation the rate enhancement was a factor of 36 , which is still substantially larger than the estimate based on experimental dispersion data, a factor of 9.6. ${ }^{33}$ Marcus and co-workers suspected the neglect of electronic polarization and intramolecular vibrations in the SPC water model ${ }^{34}$ to be responsible for an overestimation of quantum corrections. ${ }^{33}$ The SPC model was found to overestimate quantum corrections in the libration region possibly because of the too large permanent dipole moment that compensates for the missing electronic polarization.

Since these early studies a number of polarizable water models have been developed based on polarizable atomic dipoles ${ }^{35-39}$ Drude oscillator or shell models,${ }^{40-43}$ dynamically fluctuating charges ${ }^{39}$ and multistate empirical valence bond. ${ }^{44}$ The purpose of the present work is to calculate solvent reorganization and quantum corrections 
for two of these recent models, AMOEBA ${ }^{35}$ and SWM4-NDP, ${ }^{43}$ and to compare the results to earlier polarizable $\left(\mathrm{POL}_{3}{ }^{45}\right.$ ) and nonpolarizable (TIP3P, ${ }^{46} \mathrm{TIP} 4 \mathrm{P},{ }^{46} \mathrm{SPC} / \mathrm{E}^{47}$ ) water models and to the recently developed flexible $\mathrm{SPC} / \mathrm{Fw}^{48}$ model. The AMOEBA model uses a polarizable atomic multipole description of electrostatic interactions while SWM4-NDP includes polarization via classical Drude oscillators. The reaction under investigation is a prototype electron self-exchange reaction between $\mathrm{Ru}$ ions:

$$
\mathrm{Ru}^{2+}+\mathrm{Ru}^{3+*} \longrightarrow \mathrm{Ru}^{3+}+\mathrm{Ru}^{2+*}
$$

The solute, defined by the two Ru ions and the 12 first shell water molecules, is treated in all simulations with a uniform nonpolarizable representation. We have chosen to study this reaction because in previous work we have carried out ab-initio molecular dynamics simulations of solutions of the isolated ions. ${ }^{22,27}$ The parameters for the classical model used herein are chosen to reproduce the structure and dynamics of the ab-initio simulations. Estimates for outer sphere reorganization that reproduce experimental data are available ${ }^{31,49}$ and found to be similar to estimates for the isoelectronic ferrous-ferric self-exchange reaction ${ }^{31}$ (see table 1 ).

This paper is organized as follows: We start with the definition of the reorganization free energy and the quantum correction in terms of the spectral density function. Then the molecular model and the simulation protocol is described. After presenting radial distribution functions for all solvent models, we discuss the computed solvent reorganization free energies and quantum corrections in the context of previous simulation results. Finally we show that finite size effects for the self-exchange reaction Eq. 2 are small in contrast to what was found for ion oxidation. $^{7}$

\section{Theory}

Quantum and classical nonadiabatic expressions for the rate constant of electron transfer reactions have been reviewed in Ref. 33. Describing the electron transfer by two electronic states coupled to a harmonic bath (SpinBoson Hamiltonian) and adopting the saddle point approximation, one obtains the following expression for the nonadiabatic quantum reaction rate of an electron self-exchange reaction: ${ }^{11,33}$

$$
k=\frac{2 \pi}{\hbar}\left|\frac{H_{\mathrm{AB}}}{2}\right|^{2}\left(4 \pi \lambda^{p} / \beta\right)^{-1 / 2} \exp \left(-\frac{\beta \lambda}{4}\right)
$$

where $H_{\mathrm{AB}}$ is the electronic coupling matrix element, $\beta=\left(k_{\mathrm{B}} T\right)^{-1}$ is the inverse temperature, and $\lambda$ and $\lambda^{p}$ are reorganization free energies expressed in terms of the spectral density function $J(\omega)$.

$$
\begin{aligned}
\lambda=\lambda^{\mathrm{q}} & =\frac{8}{\beta \hbar \pi} \int_{0}^{\infty} d \omega \frac{J(\omega)}{\omega^{2}} \tanh \left(\frac{\beta \hbar \omega}{4}\right) \\
\lambda^{p}=\lambda^{\mathrm{q}^{\prime}} & =\frac{\beta \hbar}{\pi} \int_{0}^{\infty} d \omega J(\omega) \sinh ^{-1}\left(\frac{\beta \hbar \omega}{2}\right)
\end{aligned}
$$

Note that in the quantum expression the reorganization free energy in the prefactor, $\lambda^{p}$, does not equal the one in the exponential. In the classical limit for bath modes, $\beta \hbar \omega<<1$, the reorganization free energies of Eqs. 4 and 5 reduce to the usual classical expression

$$
\lambda=\lambda^{\mathrm{cl}}=\frac{2}{\pi} \int_{0}^{\infty} d \omega \frac{J(\omega)}{\omega}
$$

and $\lambda^{p}=\lambda$. The spectral density function $J(\omega)$ can be obtained from molecular simulation as the cosine transform of the autocorrelation function $c(t)$ of the vertical energy gap $\Delta E$ sampled in the reactant state $\mathrm{A},{ }^{11,14,27}$

$$
\begin{aligned}
\frac{J(\omega)}{\omega} & =\frac{\beta}{2} \int_{0}^{\infty} d t c(t) \cos (\omega t) \\
c(t) & =\langle\delta \Delta E(0) \delta \Delta E(t)\rangle_{\mathrm{A}} \\
\Delta E\left(\mathbf{R}^{N}\right) & =E_{\mathrm{B}}\left(\mathbf{R}^{N}\right)-E_{\mathrm{A}}\left(\mathbf{R}^{N}\right)
\end{aligned}
$$

where $\delta \Delta E(t)=\Delta E(t)-\langle\Delta E\rangle_{\mathrm{A}}$ and $\langle\ldots\rangle$ denotes the usual canonical average. The energy gap $\Delta E$ is equal to the vertical potential energy difference between the two diabatic states A (product) and B (reactant). 
The classical reorganization free energy can be calculated more directly without the explicit knowledge of the spectral density function. Taking the $t=0$ limit of the inverse transform of Eq. 7 one obtains Eq. 6 and by comparison with Eq. 8

$$
\lambda^{\mathrm{cl}}=\frac{\beta}{2}\left\langle(\delta \Delta E)^{2}\right\rangle_{\mathrm{A}}
$$

Finally, due to the assumption of linear response the right-hand side of Eq 10 equals the mean gap energy.

$$
\lambda^{\mathrm{cl}}=\langle\Delta E\rangle_{\mathrm{A}}
$$

A proof of the equivalence of Eqs. 10 and 11 is given in Ref. 21.

\section{Computational Methods}

\subsection{Definition of calculated quantities}

Here we define the quantities that are computed and discussed in the subsequent sections. In the following we skip the superscript "cl" for classical quantities and denote quantum corrected quantities with the superscript "q". In practice it is useful to break down the classical total gap energy Eq. 9, the reorganization free energy Eq. 11 and the spectral density Eq. 7 into the contribution of the ions and the first coordination shell ("inner sphere") and the contribution of the remaining solvent ("outer sphere").

$$
\begin{aligned}
\Delta E & =\Delta E_{\mathrm{i}}+\Delta E_{\mathrm{o}} \\
\lambda & =\lambda_{\mathrm{i}}+\lambda_{\mathrm{o}} \\
J(\omega) & =J_{\mathrm{i}}(\omega)+J_{\mathrm{o}}(\omega)+J_{\operatorname{cross}}(\omega)
\end{aligned}
$$

Note that the energy gap autocorrelation function Eq. 8 couples inner and outer sphere contributions. Therefore a cross correlation term appears for quantities that are defined via $c(t)$ such as the spectral density or the reorganization free energy Eq. 10.

The outer sphere contribution to the gap energy, $\Delta E_{0}$, is calculated for a given atomic configuration $\mathbf{R}^{N}$ as follows,

$$
\begin{aligned}
\Delta E_{\mathrm{o}}\left(\mathbf{R}^{N}\right) & =E_{\mathrm{B}}\left(\mathbf{R}^{N}\right)-E_{\mathrm{A}}\left(\mathbf{R}^{N}\right)-\left(I E_{1}\left(\mathbf{R}^{N}\right)-I E_{2}\left(\mathbf{R}^{N}\right)\right) \\
I E_{I}\left(\mathbf{R}^{N}\right) & =\sum_{j \neq k \in I} \frac{q_{j, \mathrm{O}} q_{k, \mathrm{O}}-q_{j, \mathrm{R}} q_{k, \mathrm{R}}}{R_{j k}}
\end{aligned}
$$

where $E_{\mathrm{A}}$ and $E_{\mathrm{B}}$ are the total electrostatic potential energy of the solvated ion pair in state $\mathrm{A}, \mathrm{Ru}^{2+} \mathrm{Ru}^{3+*}$, and state $\mathrm{B}, \mathrm{Ru}^{3+} \mathrm{Ru}^{2+*}$, respectively. The gap energies are calculated in periodic boundary conditions, unless stated otherwise. The outer sphere contribution is obtained by subtracting the self-contribution of the ionizable atoms of the two hexa-hydrates, $I E_{1}\left(\mathbf{R}^{N}\right)-I E_{2}\left(\mathbf{R}^{N}\right)$, from the total gap energy $E_{\mathrm{B}}-E_{\mathrm{A}} . I E_{1}\left(\mathbf{R}^{N}\right)$ is the difference in electrostatic potential energy of $\mathrm{Ru}\left(\mathrm{H}_{2} \mathrm{O}\right)_{6}$ in the oxidized $(\mathrm{O})$ and reduced state $(\mathrm{R})$, and $I E_{2}$ is the corresponding energy difference for $\mathrm{Ru}^{*}\left(\mathrm{H}_{2} \mathrm{O}\right)_{6}$ at the configuration sampled in aqueous solution. These self-terms which correspond to the inner sphere contribution are replaced by quantum mechanical energies, see Eq. 21 below. In Eq. $16 q_{j, \mathrm{O}}, q_{k, \mathrm{R}}$ denote atomic charges in oxidized and reduced state and $R_{j k}$ is the interatomic distance.

The outer sphere gap energies are sampled in state A at room temperature using molecular dynamics simulation. The reorganization free energy is then obtained from the average energy gap,

$$
\begin{aligned}
\lambda_{\mathrm{o}} & =\left\langle\Delta E_{\mathrm{o}}\right\rangle_{\mathrm{A}} \\
\lambda_{\mathrm{o}}^{\mathrm{f}} & =\left\langle\Delta E_{\mathrm{o}}^{\mathrm{f}}\right\rangle_{\mathrm{A}} \\
\lambda_{\mathrm{o}}^{\mathrm{i}} & =\left\langle\Delta E_{\mathrm{o}}^{\mathrm{i}}\right\rangle_{\mathrm{A}}
\end{aligned}
$$

where $\Delta E_{\mathrm{o}}=\Delta E_{\mathrm{o}}^{\mathrm{f}}+\Delta E_{\mathrm{o}}^{\mathrm{i}} . \Delta E_{\mathrm{o}}^{\mathrm{f}}$ is the fixed charge (or fixed multipole) contribution to $\Delta E_{\mathrm{o}}$, that is the contribution of the permanent charges or multipoles of the outer sphere medium. $\Delta E_{\mathrm{o}}^{\mathrm{i}}$ is the induced dipole contribution to $\Delta E_{\mathrm{o}}$ arising from all interactions involving induced dipoles. It is equal to zero if a nonpolarizable solvent model is used. 
Phenomenologically, the outer sphere contribution can be obtained from the spectral density function of the solvent expressed in terms of the frequency dependent dielectric constant.

$$
J_{\mathrm{o}}=(\Delta e)^{2}\left(\frac{1}{2 a_{1}}+\frac{1}{2 a_{2}}-\frac{1}{R}\right) \frac{\operatorname{Im} \epsilon(\omega)}{|\epsilon(\omega)|^{2}}
$$

Insertion into Eq. 6 gives $\lambda_{\mathrm{o}}$. In Eq. $20 a_{1}, a_{2}$ are the cavity radii of the two ions separated by the distance $R$, $\operatorname{Im} \epsilon(\omega)$ denotes the imaginary part of the frequency dependent dielectric constant $\epsilon(\omega)$, and $\Delta e$ is the difference of charge of the two ions in product and reactant state, $\Delta e=1$. An outline of a derivation of Eq. 20 is given in Ref. 33.

The inner sphere contribution, $\lambda_{\mathrm{i}}$ in Eq. 13, is obtained from density functional calculations at the BLYP/ LANL2DZ level of theory using the Gaussian program package. ${ }^{50}$

$$
\lambda_{\mathrm{i}}=E_{\mathrm{R}}^{*}-E_{\mathrm{R}}+E_{\mathrm{O}}^{*}-E_{\mathrm{O}}
$$

where $E_{R}$ and $E_{\mathrm{O}}$ are the potential energies at the minimum energy configuration of the isolated $\mathrm{Ru}(\mathrm{II})\left(\mathrm{H}_{2} \mathrm{O}\right)_{6}$ and $\mathrm{Ru}(\mathrm{III})\left(\mathrm{H}_{2} \mathrm{O}\right)_{6}$ complexes in vacuum, respectively, and $E_{\mathrm{R}}^{*}$ and $E_{\mathrm{O}}^{*}$ are the potential energies of the two hexa-hydrates at the minimum energy configuration of oxidized and reduced states, respectively. Alternatively, assuming that inner sphere reorganization is only due to the breathing modes of the two hexa-hydrates, $\tilde{\nu}_{\mathrm{R}}$ and $\tilde{\nu}_{\mathrm{O}}$, inner sphere reorganization can be estimated by ${ }^{33}$

$$
\lambda_{\mathrm{i}}=6 m \omega_{0}^{2} \Delta d_{0}^{2}
$$

where $m$ is the reduced mass, $\omega_{0}=(8)^{1 / 2} \pi c \tilde{\nu}_{\mathrm{O}} \tilde{\nu}_{\mathrm{R}} /\left(\tilde{\nu}_{\mathrm{O}}^{2}+\tilde{\nu}_{\mathrm{R}}^{2}\right)^{1 / 2}$, and $\Delta d_{0}$ the difference in bond length in the oxidized and reduced state.

The outer sphere contribution to the quantum correction of the exponential part, $\Delta \lambda_{o}^{\mathrm{q}}$, is obtained from the difference of the integrals Eq. 6 and Eq. 4 using the outer sphere contribution of the spectral density function, $J_{\mathrm{o}}$, instead of $J$. The corresponding contribution for the prefactor, $\Delta \lambda_{\mathrm{o}}^{\mathrm{q}^{\prime}}$, is obtained from the difference of the integrals Eq. 6 and Eq. 5.

$$
\Delta \lambda_{\mathrm{o}}^{\mathrm{q}}=\lambda_{\mathrm{o}}-\lambda_{\mathrm{o}}^{\mathrm{q}}, \quad \Delta \lambda_{\mathrm{o}}^{\mathrm{q}^{\prime}}=\lambda_{\mathrm{o}}-\lambda_{\mathrm{o}}^{\mathrm{q}^{\prime}}
$$

$J_{\mathrm{o}}$ is obtained according to Eq. 7 using molecular dynamics simulation (with the correlation function Eq. 8 calculated for the outer sphere gap energy Eq. 15), or according to Eq. 20 using experimental dielectric dispersion data. The inner sphere contribution to quantum correction is not taken from the simulations because the classical solute model was not fitted to reproduce vibrational frequencies. It is approximated by the quantum correction of the reorganization energy Eq. 22, with the effective breathing mode $\omega_{0}$ taken from experiment.

$$
\begin{aligned}
\Delta \lambda_{\mathrm{i}}^{\mathrm{q}} & =\lambda_{\mathrm{i}}\left[1-4 /\left(\beta \hbar \omega_{0}\right) \tanh \left(\beta \hbar \omega_{0} / 4\right)\right] \\
\Delta \lambda_{\mathrm{i}}^{\mathrm{q}^{\prime}} & =\lambda_{\mathrm{i}}\left[1-\beta \hbar \omega_{0} /\left(2 \sinh \left(\beta \hbar \omega_{0} / 2\right)\right)\right]
\end{aligned}
$$

Neglecting the small correction to the cross correlation in Eq. 14, the total quantum correction is the sum of the inner and outer sphere corrections.

$$
\Delta \lambda^{\mathrm{q}}=\Delta \lambda_{\mathrm{i}}^{\mathrm{q}}+\Delta \lambda_{\mathrm{o}}^{\mathrm{q}}, \quad \Delta \lambda^{\mathrm{q}^{\prime}}=\Delta \lambda_{\mathrm{i}}^{\mathrm{q}^{\prime}}+\Delta \lambda_{\mathrm{o}}^{\mathrm{q}^{\prime}}
$$

The total quantum corrected reorganization free energy of the exponential part, $\lambda^{\mathrm{q}}$, and the corresponding outer sphere contribution, $\lambda_{o}^{\mathrm{q}}$, are then given by

$$
\begin{aligned}
& \lambda^{\mathrm{q}}=\lambda_{\mathrm{i}}+\lambda_{\mathrm{o}}-\Delta \lambda^{\mathrm{q}}+\Delta \lambda_{\mathrm{fs}} \\
& \lambda_{\mathrm{o}}^{\mathrm{q}}=\lambda_{\mathrm{o}}-\Delta \lambda_{\mathrm{o}}^{\mathrm{q}}+\Delta \lambda_{\mathrm{fs}}
\end{aligned}
$$

where $\Delta \lambda_{\mathrm{fs}}$ is a finite size correction, which is added when the outer sphere reorganization free energy is estimated by molecular dynamics simulation. 


\subsection{Molecular Model}

Molecular dynamics simulations of the solvated ion pair $\mathrm{Ru}(\mathrm{II})\left(\mathrm{H}_{2} \mathrm{O}\right)_{6}-\mathrm{Ru}(\mathrm{III})\left(\mathrm{H}_{2} \mathrm{O}\right)_{6}$ were carried out with the AMBER9 ${ }^{51}$ simulation package for all water models. Ru-water interactions were modeled with harmonic bond and bending terms. Equilibrium bond lengths and force constants were chosen to reproduce the first shell coordination structure of the isolated $\mathrm{Ru}(\mathrm{II})$ and $\mathrm{Ru}(\mathrm{III})$ aqua-ions as obtained from Car-Parrinello molecular dynamics simulation. ${ }^{27}$ The intramolecular geometry and Lennard-Jones parameters of the first shell ligands were taken from the TIP3P water model. Atomic charges for $\mathrm{Ru}$ and first shell water molecules were obtained from DFT calculations of the isolated $\mathrm{Ru}(\mathrm{II})$ and $\mathrm{Ru}(\mathrm{III})$ hexahydrates in the gas phase. The electrostatic potential was calculated at the PBE/LANL2DZ level of theory and used to generate atomic RESP charges, which are summarized in table 2. The DFT calculation was carried out with Gaussian, ${ }^{50}$ the charge parametrization with the antechamber program included in the AMBER9 package. In the molecular simulations non-bonded 1-2 and 1-3 interactions were excluded, and 1-4 interactions were scaled by a factor $1 / 1.2$.

The two hexa-hydrates were placed in a cubic unit cell at a $\mathrm{Ru}-\mathrm{Ru}$ distance of $5.5 \AA$ and solvated with 499 water molecules (total number of water molecules $=511$ ). A value of $5.32 \AA$ was obtained for ferrous-ferric self-exchange by fitting experimental and calculated reorganization free energy of first row transition metal aquo-complexes. ${ }^{52}$ We have taken this latter value and added $0.18 \AA$ to account for the larger metal-oxygen distance of the Ru-ions. A number of water models are investigated: popular nonpolarizable and rigid models such as TIP3P ${ }^{46}$ TIP $4 \mathrm{P}^{46}$ and $\mathrm{SPC} / \mathrm{E},{ }^{47}$ the flexible and nonpolarizable $\mathrm{SPC} / \mathrm{Fw}$ model,${ }^{48}$ the rigid and polarizable POL3 ${ }^{45}$ and $\mathrm{SWM} 4-\mathrm{NDP}^{43}$ models, and the flexible and polarizable AMOEBA water model. ${ }^{35}$

There exist two slightly different versions of the original "SWM4" model: the SWM4-NDP model, that represents induced dipoles as the displacement of a negative charge, ${ }^{43}$ and the SWM4-DP model, that represents induced dipoles as the displacement of a positive charge. ${ }^{42}$ To account for the fact that the AMBER9 software uses point dipoles, we generate the trajectories using a "point-dipole" parametrization for which each parameter is the average of the equivalent parameters for the "negative" SWM4-NDP parametrization and the "positive" SWM4-DP parametrization. This interpolated model yields the correct liquid density and radial distribution functions that lie precisely between those of SWM4-NDP and SWM4-DP models. The energy gap calculations are then carried out with the SWM4-NDP model for configurations taken from this trajectory.

In all simulations the same nonpolarizable solute model described above is employed. In case of polarizable solvent the polarizabilities of solute atoms (ions and first-shell water molecules) were set equal to zero. The Lennard-Jones interactions between solute and solvent atoms were obtained according to the Lorentz-Berthelot mixing rules except for the AMOEBA solvent model. For the latter the original buffered 14-7 potential had to be used for solute and solvent atoms in order to obtain stable solvation of the solute $\left(R_{0}=2.316 \AA, \epsilon=0.05 \mathrm{kcal} / \mathrm{mol}\right.$ for $\mathrm{Ru}$, default values for water atoms). The change of the van der Waals parameters of the solute atoms did not affect the solute structure significantly because of the bonded description of Ru-O interactions. Solute O-H bonds kept rigid in all other solvent models were treated as flexible when the AMOEBA water model was used. The recent implementation of the AMOEBA model in the AMBER9 package was tested by simulating pure liquid water and comparing the radial distribution functions to the ones reported in the original paper of Ren and Ponder. ${ }^{35}$ Peak positions and heights as well as the equilibrium density were almost exactly reproduced. Models containing 37, 63, 127, 257, 1022 and 10005 TIP3P water molecules were prepared in addition to the 511 TIP3P water molecule system to study finite size effects.

\subsection{Simulation protocol}

The aqueous solutions were simulated using periodic boundary conditions and particle mesh Ewald summation of all electrostatic interactions including charge-charge, charge-dipole and dipole-dipole interactions. In all simulations of polarizable systems, the induced dipoles are calculated self-consistently using an iterative procedure. ${ }^{53}$ Lennard-Jones interactions are truncated at $10 \AA$. For the 37 and 63 water molecule systems a small cutoff of 4.8 and $5.5 \AA$ had to be chosen as the program does not allow for cutoffs larger than half of the smallest box dimension. Possible artifacts due to the small cutoff are discussed in section 4.4. The net charge of +5 is neutralized by a homogeneous background charge of opposite sign. The Ru-Ru distance was constrained to $5.5 \AA$. No other constraints were imposed, i.e., the ion pair was free to translate and rotate. The $\mathrm{O}-\mathrm{H}$ and $\mathrm{H}-\mathrm{H}$ distances were constrained for rigid water models using SHAKE. ${ }^{54}$

The aqueous solutions were equilibrated at a temperature of $300 \mathrm{~K}$ and pressure of 1 bar using the default temperature and pressure coupling schemes ${ }^{55}$ implemented in AMBER9. After equilibration of 500 ps, production 
runs in the NVT ensemble were carried out for 1 ns. Radial distribution functions and mean gap energies defined in Eqs. 17-19 were computed from 1000 equally spaced snapshots taken from this trajectory. Spectral density functions and outer sphere quantum corrections were computed from 25000 snapshots taken from a 100 ps trajectory.

\section{Results and Discussion}

\subsection{Solvation Structure}

The solvation structure of the $\mathrm{Ru}(\mathrm{II})-\mathrm{Ru}(\mathrm{III})$ ion pair is illustrated in Fig. 1 in terms of $\mathrm{Ru}(\mathrm{II})-\mathrm{O}$ and $\mathrm{Ru}(\mathrm{III})-\mathrm{O}$ radial distribution functions. For all solvent models, the peak corresponding to the first solvation shell agrees very well with the CPMD results. ${ }^{27}$ The force field parameter for the hexahydrates were indeed chosen to obtain this good agreement. Interestingly, the position of the second peak is very similar for all nonpolarizable solvent

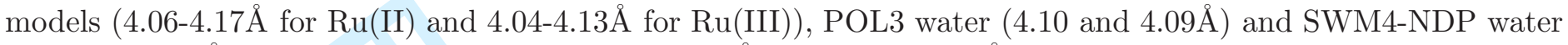
(4.06 and $4.06 \AA$ ), but not for AMOEBA water $(4.40 \AA$ for $\mathrm{Ru}(\mathrm{II}), 4.16 \AA$ for $\mathrm{Ru}(\mathrm{III})$ ). The second solvation shell is at larger distances for AMOEBA water and the distribution is significantly broader than for the other models. Interestingly, the position, height and width of the second shell radial distribution for $\mathrm{Ru}(\mathrm{II})$ is very similar to the one obtained for aqueous $\mathrm{Ca}^{2+}$ in Ref. 56. The authors noted that the Halgren function representing the van der Waals interactions in AMOEBA water slightly underestimates the interaction strength between first and second coordination shell. This is probably also the case in our simulations and the reason for the rather broad distributions observed.

Most importantly with regard to solvent reorganization, all nonpolarizable models as well as the POL3 and SWM4-NDP models respond only moderately to a change in charge mainly by narrowing the distribution of the second solvation shell. The position of the second peak is shifted to smaller distances for Ru(III) with respect to $\mathrm{Ru}$ (II) by no more than $0.05 \AA$ except in case of the SPC/E model $(0.13 \AA)$. This is in contrast to the AMOEBA water model. The change in peak position is large, $0.24 \AA$, and for $\mathrm{Ru}(\mathrm{III})$ the onset of the second peak occurs at significantly shorter distances. The explicit treatment of polarization in the AMOEBA model therefore leads to an increase in reorganization of the second solvation shell and to a significant broadening of the distributions in both oxidation states.

\subsection{Solvent reorganization free energy}

The solvent (or outer sphere) reorganization free energies, obtained from molecular dynamics simulation according to Eqs. 17-19, are summarized in table 3 for polarizable and nonpolarizable solvents. Very consistent values for $\lambda_{\mathrm{o}}$ are obtained for rigid and nonpolarizable solvent models ranging from $0.88 \mathrm{eV}$ for the TIP4P model to $1.01 \mathrm{eV}$ for the SPC/E model. The reorganization free energy increases to $1.16 \mathrm{eV}$ for the SPC/Fw model, which includes intramolecular solvent vibrations. We find that the solvent reorganization free energy does not depend on the distribution of the net charge over the solute atoms. For instance, computing the solvent gap energy once with the RESP charge model where the net charge is smeared over all solute atoms, and once for a model where the total charge is entirely localized on the $\mathrm{Ru}$ ions, we obtain virtually identical values of $0.98 \mathrm{eV}$ for TIP3P water. The potential difference at the site of the $\mathrm{Ru}$ ions is therefore a very good approximation to the potential difference averaged over all solute atoms.

The explicit treatment of electronic polarizability reduces solvent reorganization free energy by $22 \%$ for SWM4NDP water and by $11 \%$ for POL3 water relative to the TIP3P water model. An increase of $5 \%$ is obtained for AMOEBA water. The relatively small changes are in contrast to the prediction of continuum theory (Eq. 1) stating that reorganization free energy is inversely proportional to the optical dielectric constant for polar media $\left(\epsilon_{\mathrm{S}}>>1\right)$. Assuming $\epsilon_{\mathrm{op}}=1.6$ and $\epsilon_{\mathrm{s}}=80$ for the polarizable water models investigated $\left(\epsilon_{\mathrm{op}}=1.63\right.$ for SWM4$\mathrm{NDP})$, and $\epsilon_{\mathrm{s}}=92$ for TIP3P water, the reorganization free energy is predicted to decrease by a factor $(1 / 1.6-$ $1 / 80) /(1-1 / 92)=0.62(38 \%)$ according to the continuum formula.

Our results are in line with recent work of Matyushov and co-workers, who carried out Monte Carlo simulations for intramolecular charge separation and recombination. ${ }^{19}$ The authors reported a decrease in the total solvent reorganization free energy of $22 \%$ and $18 \%\left(=\lambda_{1}\right.$ and $\lambda_{2}$ in Ref. 19, respectively) when $\epsilon_{\mathrm{op}}\left(\epsilon_{\infty}\right.$ in Ref. 19) was varied from 1 to 1.755 at constant permanent dipole moment of the solvent. The reduction in reorganization free energy was in good agreement with an analytic model, but smaller than predicted by standard continuum models, $33 \%$ according to the Lippert-Mataga equation in the limit $\epsilon_{\mathrm{S}}>>1$ (see Ref. 19 for an expression), and $43 \%$ 
according to the Marcus expression Eq. 1. Note, however, that the latter is not appropriate for the intramolecular electron transfer studied in Ref. 19. Thus, the Monte Carlo simulation of Ref. 19 and our molecular dynamics simulation suggest that the dependence of solvent reorganization free energy on the optical dielectric constant is weaker than predicted by standard continuum models.

The Marcus formula Eq. 1 can be interpreted as the difference of two equilibrium solvation free energies calculated for solvation in two dielectric media with dielectric constants $\epsilon_{\mathrm{S}}$ and $\epsilon_{\mathrm{op}}$. According to Matyushov and co-workers the failure of the dielectric models is caused by the incorrect calculation of both the total solvent free energy and its electronic component. ${ }^{19}$ The combination of the two errors leads to a much stronger dependence of solvent reorganization on $\epsilon_{\mathrm{op}}$ than obtained from simulation and analytic theories of solvent reorganization.

The significant deviation in reorganization free energy for the polarizable water models requires some explanation. We note that the inclusion of explicit electronic polarizability changes both electronic response and nuclear response. In order to distinguish the two we consider the following transformations

$$
\begin{aligned}
\text { TIP3P } / / \text { TIP3P } & \rightarrow \mathrm{X} / / \text { TIP3P } \\
\mathrm{X} / / \text { TIP3P } & \rightarrow \mathrm{X} / / \mathrm{X}
\end{aligned}
$$

where X denotes a polarizable water model (AMOEBA,SWM4-NDP or POL3). X//Y stands for the mean energy gap $\left(=\lambda_{\mathrm{o}}\right.$, Eq. 17) calculated with the potential energy function of water model $\mathrm{X}$ for solvent configurations sampled with the potential energy function of water model Y. We have chosen the commonly used TIP3P model as the reference nonpolarizable water model Y. The first transformation Eq. 28 is the change in $\lambda_{\mathrm{o}}$ due to the different parametrization of the electrostatic energy terms of water model X wrt TIP3P water (permanent charges of X are smaller than for TIP3P and inducible dipoles are included). Note that the solvent configurations for which the gap energies of $\mathrm{X}$ are calculated remain unchanged and are the ones from the TIP3P equilibrium trajectory. In the second transformation Eq. 29 the change in $\lambda_{\mathrm{o}}$ is due to nuclear reorganization from the equilibrium solvation structure of TIP3P water to the equilibrium solvation structure of water model X (see radial distributions in Figure 1 for the different solvation structures).

The results of our analysis are summarized in table 4 . We find that the mean gap energies X//TIP3P are indeed reduced by $34 \%$ (X=AMOEBA), $29 \%$ (X=SWM4-NDP), and 26\% (X=POL3) compared to TIP3P//TIP3P (Eq. 28). Interestingly, for AMOEBA water this decrease in reorganization free energy is close to the $38 \%$ reduction suggested by the ratio of Pekar factors $\left[(1 / 1.6-1 / 81) /(1-1 / 92)=0.62\right.$, assuming $\epsilon_{\mathrm{op}}=1.6$ for AMOEBA water]. For POL3 water the decresase is smaller, which is not unexpected as POL3 water seems to have the tendency to underestimate electronic polarization effects. ${ }^{35}$ Breaking down the solvent reorganization free energy in the contributions of the fixed water dipoles, $\lambda_{\mathrm{o}}^{\mathrm{f}}$, and the induced dipoles, $\lambda_{\mathrm{o}}^{\mathrm{i}}$, we find that both contribute to reduction in reorganization free energy. The decrease in fixed dipole contribution correlates well with the reduction of the permanent dipole moment of the polarizable models: the permanent dipole moment of AMOEBA, SWM4-NDP and POL3 is scaled by a factor of $0.75,0.79$, and 0.86 relative to TIP3P giving a decrease in the fixed dipole contribution to reorganization free energy by a factor of $0.81,0.83$ and 0.86 (14-19\%). The factor 0.81 for AMOEBA includes the fixed quadrupolar contribution. The induced dipole contribution does not compensate for the loss in fixed dipole contribution but reduces reorganization free energy even further. Remarkably consistent values are obtained for the three water models, $\lambda_{\mathrm{o}}^{\mathrm{i}}=-0.11$ to $-0.14 \mathrm{eV}$, corresponding to a decrease in reorganization free energy of $11-14 \%$.

"Relaxing" the solvation structure by going from the ensemble of equilibrium configurations of the TIP3P model to the ensemble of equilibrium configurations of the polarizable water model (Eq. 29), we obtain an increase in reorganization free energy that strongly depends on the polarizable water model used: $39 \%$ for AMOEBA, $15 \%$ for POL3 and $7 \%$ for SWM4-NDP. This increase can not be explained by continuum theory because the static dielectric constant is similar for all polarizable models and TIP3P water and the nuclear response should therefore also be similar. Rather, it is a feature of the particular atomistic model used to describe solute-solvent interactions. The large increase in reorganization for AMOEBA water is a consequence of the much stronger ionic response in the second solvation shell of the ions, when compared to TIP3P water (compare radial distributions of $\mathrm{Ru}^{2+}$ and $\mathrm{Ru}^{3+}$ in Fig. 1 and discussion in section 4.1). The corresponding increase in reorganization free energy compensates for the reduction in reorganization free energy observed for transformation Eq. 28, thereby shifting the final solvent reorganization free energy of AMOEBA water $5 \%$ beyond the original value of the TIP3P model. The smaller increase in reorganization free energy for POL3 and SWM4-NDP water is consistent with the smaller deviations in radial distribution functions relative to the TIP3P model. Consequently, a net reduction in reorganization free energy of 11 and $22 \%$ remains for these two water models. 
Correcting for the finite size of our model solutions $(+0.07 \mathrm{eV}$, see table 6 and section 4.4$)$, our final estimates for $\lambda_{\mathrm{o}}$ range from 0.83 to $1.23 \mathrm{eV}$ for polarizable and nonpolarizable solvents, and from 0.76 to $1.06 \mathrm{eV}$ if quantum corrections are included (see section 4.3). Estimates from continuum models that reproduce experimental rate constants, $1.11^{57}$ and $1.20 \mathrm{eV},{ }^{31}$ lie in the former range of values, while the estimate based on kinetic measurements of activation enthalpy is slightly larger, $1.33 \mathrm{eV} \cdot{ }^{49}$ Our models tend to underestimate reorganization free energy, in particular the polarizable water models that are corrected for quantum effects. A possible reason could be that the actual Ru-Ru distance where ET occurs is larger than $5.5 \AA$ and closer to $6.5 \AA$ adopted in previous studies. ${ }^{31,57}$ Another reason could be that explicit electronic solute polarizability was not included in our simulations. It was shown to increase solvent reorganization free energy for charge recombination reactions. ${ }^{19}$ Finally we note that similar outer sphere reorganization free energies have been reported for Fe(II)-Fe(III) self-exchange, $1.2 \mathrm{eV}^{24}$ from ab-initio molecular dynamics simulation (after subtracting an estimate for the inner sphere contribution) and $1.20^{31}-1.50 \mathrm{eV}^{52}$ (see table 1 ).

In light of the present investigation it is rather surprising that in two previous MD simulation studies of ferrous-ferric self-exchange the solvent reorganization free energy was substantially overestimated, $2.34^{9}$ and 2.12 $2.55 \mathrm{eV} .{ }^{14}$ The solvent models used in these studies (SPC in Ref. 9 and flexible TIP3P in Ref. 14) are probably not the source for the discrepancy as their properties are similar to some of the water models used in this study. We also do not expect that the smaller metal-oxygen bond lengths and the different net charge distribution (entirely located on the metal ions in Refs.9 and 14) can have such a large effect. Moreover, our analysis of finite size effects (see section 4.4) suggests that the finite cut off used in Refs. 14 and 9 for electrostatic interactions would lead to a small underestimation, but not to an overestimation of gap energies. What might be partly responsible for the deviation with our results are differences in solvation structure of second and higher coordination shells. For instance, the position of the second peak shifts to smaller distances in response to oxidation by about 0.2$0.3 \AA$ (estimated from Figs. 2 and 3 of Refs. 14 and 9, respectively) but by less than $0.05 \AA$ in this work. This might be a consequence of different simulation protocols or of the finite cutoff used in the earlier studies for truncation of electrostatic interactions. ${ }^{9}$

\subsection{Quantum corrections}

The quantum corrections obtained from simulation are summarized in table 5 and compared to the estimate based on experimental dielectric dispersion data for pure liquid water (using Eq. 20). The outer sphere quantum correction of the exponential part is about $0.1 \mathrm{eV}$ for all solvent models investigated except for the flexible $\mathrm{SPC} / \mathrm{Fw}$ model for which we obtain a larger value of $0.17 \mathrm{eV}$. The estimates obtained from simulation compare very well with the estimate based on experimental data, $0.13 \mathrm{eV}$. Adding $0.07 \mathrm{eV}$ for the inner sphere quantum correction (effective breathing mode, only, independent on the solvent model), and accounting for the small quantum correction of the prefactor, we obtain a total ratio of $k^{\mathrm{q}} / k^{\mathrm{cl}}=4-11$ for the solvent models investigated, and a factor 7.7 from experimental dispersion data.

The frequency-resolved outer sphere quantum correction of the exponential part is illustrated in Fig. 2 (in panel A for nonpolarizable models and in panel B for polarizable models). As one can see in panel A the quantum corrections for the rigid nonpolarizable models are overestimated in the libration region $500-1000 \mathrm{~cm}^{-1}$ relative to the estimate based on experimental dielectric dispersion data. Interestingly, the overestimation of $\Delta \lambda_{0}^{\mathrm{q}}$ at 1000 $\mathrm{cm}^{-1}$ (panel C) compensates to a good approximation the neglect of intramolecular contributions (bending mode at $1640 \mathrm{~cm}^{-1}$, stretching modes at 3000-3600 $\mathrm{cm}^{-1}$ ) explaining the apparent good agreement with experimental data. Note that the quantum correction for the flexible nonpolarizable SPC/Fw model overshoots in the bending region causing the largest quantum correction of all solvent models investigated, $k^{\mathrm{q}} / k^{\mathrm{cl}}=10.9$. Best agreement with experiment is achieved with the AMOEBA water model, panel B. The corrections in both the libration and bending region is well reproduced. However, the contributions from the stretching regions are severely underestimated explaining the rather small total quantum correction $k^{\mathrm{q}} / k^{\mathrm{cl}}=4.2$ obtained with this model.

The quantum correction obtained for $\mathrm{Ru}(\mathrm{II})-\mathrm{Ru}(\mathrm{III})$ self-exchange, $k^{\mathrm{q}} / k^{\mathrm{cl}}=4-11$, is close to the estimate for Fe(II)-Fe(III) self-exchange $\left(9.6^{33}\right)$ but significantly smaller than the values obtained in Ref. 11 from quantum Monte Carlo simulation (65) and from the harmonic bath approximation (36). Marcus and coworkers suspected that the neglect of electronic and vibrational polarization in the SPC model of Ref. 11 leads to artifacts in the spectral density causing an overestimation of quantum corrections. Calculating the spectral density from dielectric dispersion data of Ref. 58 he showed that the spectra of the MCY and TIP4P models indeed overestimate the quantum correction in the libration region. This is also what we find in the present study for the rigid 
nonpolarizable models (Fig $2(\mathrm{~A})$ ). However, as explained above, we find that the overestimation in the libration region is compensated to a large extend by the neglect of quantum corrections in the stretching region bringing the total quantum correction close to the estimate obtained from experimental dielectric dispersion data.

Analyzing the contributions of the quantum correction we find that the inner and outer sphere contributions are $0.21 \mathrm{eV}$ and $0.20 \mathrm{eV}$ in Ref. 11 (full quantum Monte Carlo), $0.12 \mathrm{eV}$ and $0.11 \mathrm{eV}$ in Ref. 33 and 0.07 and 0.10-0.12 eV in the present study (rigid nonpolarizable models). The discrepancy for the inner sphere contribution can be explained by noting that this term was computed from a single experimental frequency (breathing mode, $\approx 400 \mathrm{~cm}^{-1}$ ) in Ref. 33 and in present work, while it was obtained from simulation in Ref. 11 using Lennard-Jones type of interactions. The estimate from simulation probably also included higher frequencies $\left(\right.$ up to $\left.\approx 1000 \mathrm{~cm}^{-1}\right)$ which led to larger quantum corrections than for the single breathing mode. The discrepancy for the outer sphere contribution is less obvious. Ultimately it must be attributed to the large spectral density obtained for the SPC model in the region $800-1000 \mathrm{~cm}^{-1}$ (Figure 1 of Ref. 11).

\subsection{Size effects}

Size effects on the outer sphere mean vertical gap energies are investigated by computing this quantity for a series of TIP3P water model systems of increasing system size. The results are summarized in table 6 . An increase of the number of solvent molecules from 37 to 10005 leads to a rather small increase of $\lambda_{\mathrm{o}}$ by $1.037-0.957=0.080 \mathrm{eV}$. The data obtained for seven system sizes were fitted to inverse powers of the box length $L$. Best correlation was obtained for the $1 / L$ fit as shown in Figure 3 . The extrapolation to the infinite dilution limit $L \rightarrow \infty$ gives a value of $1.052 \mathrm{eV}$, just $0.07 \mathrm{eV}$ higher than the $0.98 \mathrm{eV}$ of the 511 water molecule system used in all previous discussions.

The weak size dependence observed for electron transfer is in strong contrast to the large size dependence of more than $1 \mathrm{eV}$ reported for the solvent reorganization free energy for oxidation of a single Fe(II)-ion. ${ }^{7}$ This is not unexpected because in ET reactions the solvent responds to a change in the solute dipole rather than to a change in total solute charge. However, one would then also expect that the size dependence for ET decays faster than for oxidation, i.e. faster than $1 / L .^{7}$

One might suspect the periodic boundary conditions to be partly responsible for the weak size dependence. This was in fact the case for the weak size dependence of the free energy for charging a Lennard-Jones particle. ${ }^{59}$ However, computing the reorganization free energy of the solvent in the central cell only (i.e. using cluster boundary conditions, column "Cluster" in table 6) we obtain similar albeit slightly smaller values than with periodic boundary conditions (column "PBC" in table 6). The effect of the periodic images is a small increase in outer sphere reorganization free energy of not more than $0.15 \mathrm{eV}$ for the 37 and 63 water molecule systems. The effect is less than $0.02 \mathrm{eV}$ and within the statistical uncertainty for the solutions containing 511 and more water molecules.

In the 37 water molecule system each of the 12 first shell ligands is hydrogen bonded by 2 water molecules, and although the second solvation shell is only partly formed, the reorganization free energy is already $83 \%$ of the 10005 molecule system (in cluster boundary conditions). This implies that the dominant part of the reorganization free energy is due to the second solvation shell. Figure 4 shows that this is indeed the case. The graph shows the radial reorganization density $\rho_{\lambda}$ as a function of the distance $d$

$$
\rho_{\lambda}=\mathrm{d} \lambda_{q \mu} / \mathrm{d} d
$$

where $\lambda_{q \mu}$ is the solvent reorganization free energy obtained from the charge-dipole interactions between the solute and TIP3P water. Integration over $\rho_{\lambda}$ gives the total outer sphere reorganization free energy $\lambda_{q \mu}$ (inset in Figure 4 ) which is to a very good approximation equal to $\lambda_{0}$. The distance $d$ is defined as the shortest of the two distances between a given water molecule and the two Ru-ions. The density $\rho_{\lambda}$ measures the reorganization free energy in (non-spherical) shells at a distance $d$ to any of the two ions.

The radial density exhibits a large positive peak at about $4.1 \AA$ and a smaller negative peak at $4.5 \AA$. The positive part is due to the first shell oxygen atoms generating a positive gap energy and the negative part is due to the first shell hydrogen atoms generating a smaller contribution of opposite sign. The former is almost size independent in strong contrast to the latter, which becomes significantly smaller for smaller system sizes. The net effect is that after integration over the second solvation shell the reorganization free energy of the 37 water molecule system is $0.26 \mathrm{eV}$ higher than for the 10005 water molecule system ("2nd shell" in table 6), thereby partly compensating for the absence of reorganization free energy of higher solvation shells. The apparent large 
reorganization free energy of the small water molecule systems is therefore due to a change in orientation of second shell water dipoles which effectively leads to an increase in gap energy. This is probably a consequence of both the artificial interactions at the boundary of these small systems and the small cutoff that had to be used to truncate Lennard-Jones interactions (required to be smaller than half the box dimension in AMBER). Returning to table 6 we observe that the second and third shell contributions are converged within the statistical uncertainties for the 511 water molecule system. The contributions of higher solvation shells seem to be well converged for the 1022 molecule system at least when compared to the infinite dilution limit obtained from the extrapolation against $1 / L$.

\section{Conclusion}

In the present study we have computed the solvent reorganization free energy for electron self-exchange of a nonpolarizable solute in nonpolarizable and polarizable solvents. We find that inclusion of explicit electronic solvent polarizability introduces two opposing effects. Firstly, a decrease in reorganization free energy due to a decrease in electronic response. This is a consequence of the decrease of the large effective dipole moment of nonpolarizable water models to the smaller gas-phase like fixed dipole/multipole moment in polarizable models. The induced dipole contribution does not compensate for this loss, but tends to reduce reorganization free energy even further. Secondly, we observe an increase in reorganization free energy due to an increased nuclear response. This is due to the stronger change in solvation structure upon electron transfer in polarizable water models. The sum of these opposing effects give a decrease in reorganization free energy that is smaller than predicted by continuum theory. Thus our simulations confirm the view that standard continuum models predict a too strong dependence of solvent reorganization free energy on the optical dielectric constant. ${ }^{19}$

The varying degree of reduction in reorganization free energy for polarizable models was explained by the different increase of structural response relative to TIP3P. The difference of the radial distribution function for second shell water molecules of $\mathrm{Ru}^{2+}$ and $\mathrm{Ru}^{3+}$ is much stronger for AMOEBA water than for TIP3P water. This leads to a strong increase in reorganization free energy for AMOEBA water that effectively cancels the reduction in reorganization free energy caused by the smaller permanent dipole moment. For SWM4-NDP and POL3 the solvation structure of the two ions is similar to TIP3P water, and a significant reduction in reorganization free energy due to the reduced electronic response remains. Differences in solvation structure might explain why for certain polarizable water models the total reorganization is significantly reduced ${ }^{10,20}$ or remains almost unchanged. ${ }^{6,13,15}$

Using the harmonic bath model we find that the solvent nuclear quantum effects are not overestimated in standard rigid nonpolarizable water models as suggested in Ref. 33. The quantum corrections in the libration modes are indeed overestimated but the overestimation compensates to a good approximation for the neglect of corrections in the stretching modes. The polarizable models reproduce the quantum corrections of the libration modes very well but significantly underestimate the contributions in the stretching region. The total quantum corrections for the nonpolarizable models are therefore slightly larger than for the polarizable models and in better agreement with estimates based on dielectric dispersion data. The discrepancy of inner sphere nuclear quantum corrections in Ref. 11 and Ref. 33 (and present work) is in part caused by the different treatment of inner sphere vibrational modes. The deviation in outer sphere quantum correction is less obvious since all rigid nonpolarizable water models investigated give quantum corrections that are smaller than in Ref. 11 and in better agreement with estimates based on experimental data.

Finally we find that the reorganization free energy for self-exchange between two Fe-like ions is only weakly dependent on system size. The dependence is tentatively proportional to $1 / L$ attenuated by a small prefactor. Ab-initio molecular dynamics simulation of electron transfer reactions ${ }^{24}$ should therefore suffer considerably less from finite size effects than ab-initio simulations of oxidation reactions. ${ }^{7,27}$ Yet a formulation of an analytic size correction similar to the one for oxidation ${ }^{7}$ would be desirable in order to confirm and understand the size dependence observed in present simulation.

\section{Acknowledgments}

We want to thank Dr. P. Ren and Dr. J. W. Ponder for providing a modified version of the analyze program of the AMOEBA simulation package. J. B. acknowledges financial support from EPSRC and The Royal Society 
for a University Research Fellowship, and access to a compute cluster at the Center of Computational Chemistry, University of Cambridge.

\section{References}

[1] Marcus, R. A. J. Chem. Phys., 1956, 24, 966.

[2] Marcus, R. A. Discuss. Faraday Soc., 1960, 29, 21.

[3] Marcus, R. A. J. Chem. Phys., 1965, 43, 679.

[4] Marcus, R. A.; Sutin, N. Biochim. Biophys. Acta, 1985, 811, 265.

[5] Marcus, R. A. Rev. Mod. Phys., 1993, 65, 599.

[6] Bader, J. S.; Cortis, C. M.; Berne, B. J. J. Chem. Phys., 1997, 106, 2372.

[7] Ayala, R.; Sprik, M. J. Phys. Chem. B, 2007, p asap.

[8] Warshel, A. J. Phys. Chem., 1982, 86, 2218.

[9] Kuharski, R. A.; Bader, J. S.; Chandler, D.; Sprik, M.; Klein, M. L.; Impey, R. W. J. Chem. Phys., 1988, 89,3248 .

[10] King, G.; Warshel, A. J. Chem. Phys., 1990, 93, 8682.

[11] Bader, J. S.; Kuharski, R. A.; Chandler, D. J. Chem. Phys., 1990, 93, 230.

[12] Marchi, M.; Gehlen, J. N.; Chandler, D.; Newton, M. D. J. Am. Chem. Soc., 1993, 115, 4178.

[13] Calhoun, A.; Voth, G. A. J. Phys. Chem. B, 1998, 102, 8563.

[14] Ando, K. J. Chem. Phys., 2001, 114, 9470.

[15] Ando, K. J. Chem. Phys., 2001, 115, 5228.

[16] Hartnig, C.; Koper, M. T. M. J. Chem. Phys., 2001, 115, 8540.

[17] Small, D. W.; Matyushov, D. V.; Voth, G. A. J. Am. Chem. Soc., 2003, 125, 7470.

[18] Leontyev, I. V.; Basilevsky, M. V.; Newton, M. D. Theor. Chem. Acc., 2004, 111, 110.

[19] Gupta, S.; Matyushov, D. V. J. Phys. Chem. A, 2004, 108, 2087.

[20] Ceccarelli, M.; Marchi, M. J. Phys. Chem. B, 2003, 10\%, 5630.

[21] Tateyama, Y.; Blumberger, J.; Sprik, M.; Tavernelli, I. J. Chem. Phys., 2005, 122, 234505.

[22] Blumberger, J.; Sprik, M. J. Phys. Chem. B, 2005, 109, 6793.

[23] Vener, M. V.; Tovmash, A. V.; Rostov, I. V.; Basilevski, M. V. J. Phys. Chem. B, 2006, 110, 14950.

[24] Sit, P. H.-L.; Cococcioni, M.; Marzari, N. Phys. Rev. Lett., 2006, 97, 028303.

[25] Blumberger, J.; Klein, M. L. J. Am. Chem. Soc., 2006, 128, 13854.

[26] Blumberger, J.; Tavernelli, I.; Klein, M. L.; Sprik, M. J. Chem. Phys., 2006, 124, 64507.

[27] Blumberger, J.; Sprik, M. Theor. Chem. Acc., 2006, 115, 113.

[28] Leontyev, I. V.; Tachiya, M. J. Chem. Phys., 2007, 126, 064501.

[29] Sulpizi, M.; Raugei, S.; VandeVondele, J.; Carloni, P.; Sprik, M. J. Phys. Chem. B, 2007, 111, 3969. 
[30] Tateyama, Y.; Blumberger, J.; Ohno, T.; Sprik, M. J. Chem. Phys., 2007, 126, 204506.

[31] Brunschwig, B. S.; Creutz, C.; McCartney, D. H.; Sham, T.-K.; Sutin, N. Faraday Discuss. Chem. Soc., 1982, 74, 113.

[32] Rick, S. W.; Stuart, S. J.; Berne, B. J. J. Chem. Phys., 1994, 101, 6141.

[33] Song, X.; Marcus, R. A. J. Chem. Phys., 1993, 99, 7768.

[34] Berendsen, H. J. C.; Postma, J. P.; van Gunsteren, W. F.; Hermans, J. in Intermolecular Forces, Pullmann, B., Ed., p 331. Reidel, Dordrecht, 1981.

[35] Ren, P.; Ponder, J. W. J. Phys. Chem. B, 2003, 107, 5933.

[36] Paricaud, P.; Predota, M.; Chialvo, A. A.; Cummings, P. T. J. Chem. Phys., 2005, 122, 244511.

[37] Burnham, C. J.; Xantheas, S. S. J. Chem. Phys., 2002, 116, 5115.

[38] Fanourgakis, G. S.; Xantheas, S. S. J. Phys. Chem. A, 2006, 110, 4100.

[39] Stern, H. A.; Rittner, F.; Berne, B. J.; Friesner, R. A. J. Chem. Phys., 2001, 115, 2237.

[40] van Maaren, P. J.; van der Spoel, D. J. Phys. Chem. B, 2001, 105, 2618.

[41] Yu, H.; van Gunsteren, W. F. J. Chem. Phys., 2004, 121, 9549.

[42] Lamoureux, G.; MacKerell Jr., A. D.; Roux, B. J. Chem. Phys., 2003, 119, 5185.

[43] Lamoureux, G.; Harder, E.; Vorobyov, I. V.; Roux, B.; MacKerell Jr, A. D. Chem. Phys. Lett., 2005, 418, 241.

[44] Jeon, J.; Lefohn, A. E.; Voth, G. A. J. Chem. Phys., 2003, 118, 7504.

[45] Caldwell, J. W.; Kollman, P. A. J. Phys. Chem., 1995, 99, 6208.

[46] Jorgensen, W. L.; Chandrasekhar, J.; Madura, J. D.; Impey, R. W.; Klein, M. L. J. Chem. Phys., 1983, 79, 926.

[47] Berendsen, H. J. C.; Grigera, J. R.; Straatsma, T. P. J. Phys. Chem., 1987, 91, 6269.

[48] Wu, Y.; Tepper, H. L.; Voth, G. A. J. Chem. Phys., 2006, 124, 024503.

[49] Bernhard, P.; Helm, L.; Ludi, A.; Merbach, A. E. J. Am. Chem. Soc., 1985, 107, 312.

[50] Gaussian 03, Revision C.01, M. J. Frisch, G. W. Trucks, H. B. Schlegel, G. E. Scuseria, M. A. Robb, J. R. Cheeseman, J. A. Montgomery, Jr., T. Vreven, K. N. Kudin, J. C. Burant, J. M. Millam, S. S. Iyengar, J. Tomasi, V. Barone, B. Mennucci, M. Cossi, G. Scalmani, N. Rega, G. A. Petersson, H. Nakatsuji, M. Hada, M. Ehara, K. Toyota, R. Fukuda, J. Hasegawa, M. Ishida, T. Nakajima, Y. Honda, O. Kitao, H. Nakai, M. Klene, X. Li, J. E. Knox, H. P. Hratchian, J. B. Cross, C. Adamo, J. Jaramillo, R. Gomperts, R. E. Stratmann, O. Yazyev, A. J. Austin, R. Cammi, C. Pomelli, J. W. Ochterski, P. Y. Ayala, K. Morokuma, G. A. Voth, P. Salvador, J. J. Dannenberg, V. G. Zakrzewski, S. Dapprich, A. D. Daniels, M. C. Strain, O. Farkas, D. K. Malick, A. D. Rabuck, K. Raghavachari, J. B. Foresman, J. V. Ortiz, Q. Cui, A. G. Baboul, S. Clifford, J. Cioslowski, B. B. Stefanov, G. Liu, A. Liashenko, P. Piskorz, I. Komaromi, R. L. Martin, D. J. Fox, T. Keith, M. A. Al-Laham, C. Y. Peng, A. Nanayakkara, M. Challacombe, P. M. W. Gill, B. Johnson, W. Chen, M. W. Wong, C. Gonzalez, and J. A. Pople, Gaussian, Inc., Wallingford CT, 2004.

[51] Case, D.A.; Darden, T.A.; Cheatham, III, T.E.; Simmerling, C.L.; Wang, J.; Duke, R.E.; Luo, R.; Merz, K.M.; Pearlman, D.A.; Crowley, M.; Walker, R.C.; Zhang, W.; Wang, B.; Hayik, S.; Roitberg, A.; Seabra, G.; Wong, K.F.; Paesani, F.; Wu, X.; Brozell, S.; Tsui, V.; Gohlke, H.; Yang, L.; Tan, C.; Mongan, J.; Hornak, V.; Cui, G.; Beroza, P.; Mathews, D.H.; Schafmeister, C.; Ross, W.S. and Kollman, P.A. (2006), AMBER 9, University of California, San Francisco. 
[52] Rosso, K. M.; Rustad, J. R. J. Phys. Chem. A, 2000, 104, 6718.

[53] Toukmaji, A.; Sagui, C.; Board, J.; Darden, T. J. Chem. Phys., 2000, 113, 10913.

[54] Andersen, H. C. J. Comput. Phys., 1983, 52, 24.

[55] Berendsen, H. J. C.; Postma, J. P. M.; van Gunsteren, W. F.; DiNola, A.; Haak, J. R. J. Chem. Phys., 1984, 81,3684 .

[56] Piquemal, J.-P.; Perera, L.; Cisneros, G. A.; Ren, P.; Pedersen, L. G.; Darden, T. A. J. Chem. Phys., 2006, $125,054511$.

[57] Rotzinger, F. P. J. Chem. Soc. Dalton Trans., 2002, p 719.

[58] Neumann, M. J. Chem. Phys., 1986, 85, 1567.

[59] Hummer, G.; Pratt, L. R.; Garcia, A. E. J. Phys. Chem. A, 1998, 102, 7885.

[60] Kusalik, P. G.; Svishchev, I. M. Science, 1994, 265, 1219.

[61] Allen, M. P.; Tildesley, D. J., Eds. Computer Simulation of Liquids. Clarendon Press, Oxford, 2000.

[62] Bernhard, P.; Ludi, A. Inorg. Chem., 1984, 23, 870.

[63] Afsar, M. N.; Hasted, J. B. Infrared Phys., 1978, 18, 835.

[64] Hasted, J. B.; Husain, S. K.; Frescura, F. A. M.; Birch, J. R. Infrared Phys., 1987, 27, 11.

[65] Hale, G. M.; Querry, M. R. Appl. Opt., 1973, 12, 555. 
Table 1: Experimental and computed estimates for outer sphere reorganization free energies $\lambda_{\mathrm{o}}$ of $\mathrm{Ru}(\mathrm{II})-\mathrm{Ru}(\mathrm{III})$ electron self-exchange in aqueous solution (or for Fe(II)-Fe(III) self-exchange where indicated). The columns "pol" and "qc" indicate whether explicit solvent electronic polarization and quantum corrections, respectively, are included in estimates obtained from molecular simulations.

$\begin{array}{lccc} & \lambda_{\mathrm{o}}(\mathrm{eV}) & \text { pol } & \text { qc } \\ \text { Bernhard85 }^{a} & 1.33 & & \\ \text { Brunschwig82 }^{b} & 1.20 & & \\ \text { Rosso00 }^{c} & 1.50 & & \\ \text { Rotzinger02 }^{d} & 1.11 & & \\ \text { Kuharski88 }^{e} & 2.34 & \text { no } & \text { no } \\ \text { Bader90 }^{f} & 2.17 & \text { no } & \text { yes } \\ \text { Ando01 }^{g} & 2.12 & \text { no } & \text { no } \\ \text { Ando01 }^{h} & 2.55 & \text { no } & \text { no } \\ \text { Sit06 }^{i} & 1.2 & \text { yes } & \text { no } \\ \text { this work }^{j} & 0.95-1.23 & \text { no } & \text { no } \\ & 0.85-1.06 & \text { no } & \text { yes } \\ & 0.83-1.10 & \text { yes } & \text { no } \\ & 0.76-1.02 & \text { yes } & \text { yes }\end{array}$

${ }^{a}$ Ref. 49. Obtained from kinetic measurements of $\Delta H^{\ddagger}$. Outer sphere activation entropy is assumed to be zero, inner sphere contribution is estimated from harmonic force constants and change of bond lengths. ${ }^{b}$ Ref. 31 . Eq. 1 for ion-ion distance $R=6.5 \AA .{ }^{c}$ Ref. 52 . Eq. 1 for Fe(II)-Fe(III) self-exchange at $R=5.25 \AA .{ }^{d}$ Ref. 57 . Combined quantum mechanical/continuum calculation. Value corresponds to $\lambda-\lambda_{\text {in }}$ at $R=6 \AA$, where $\lambda$ was taken from table 8 and $\lambda_{\text {in }}$ from table 3 of Ref. 57. ${ }^{e}$ Ref. 9. MD simulation of $\mathrm{Fe}(\mathrm{II})-\mathrm{Fe}(\mathrm{III})$ self-exchange using the SPC water model, $R=5.5 \AA$. Value corresponds to contribution of second shell and remaining solvent. ${ }^{f}$ Ref. 11 . Pathintegral simulation of $\mathrm{Fe}(\mathrm{II})-\mathrm{Fe}(\mathrm{III})$ self-exchange using the SPC water model, $R=5.5 \AA$. Value corresponds to contribution of region 2 and $3 .^{g}$ Ref. 14. MD simulation of Fe(II)-Fe(III) self-exchange using the flexible TIP3P water model, $R=5 \AA .{ }^{h}$ Same as footnote $g$ but $R=6 \AA$. ${ }^{i}$ Ref. 24 . Ab-initio molecular dynamics simulation of $\mathrm{Fe}(\mathrm{II})$-Fe(III) self-exchange. The total reorganization free energy obtained, $2.0 \mathrm{eV}$, is reduced by the inner sphere contribution of $0.849 \mathrm{eV}$ taken from Ref. 57. ${ }^{j}$ Eq. 17, obtained from molecular dynamics simulation of 511 water molecule systems in periodic boundary conditions. Value taken from table 3 and a finite size correction $\lambda_{\mathrm{fs}}=0.07$ $\mathrm{eV}$ is added. Quantum corrected values (qc): Eq. 27 with $\Delta \lambda_{\mathrm{o}}^{\mathrm{q}}$ taken from table 5. 
Table 2: Point charges used for $\mathrm{Ru}$ and first shell water molecules in molecular dynamics simulation of aqueous $\mathrm{Ru}(\mathrm{II})-\mathrm{Ru}(\mathrm{III})$. All values are given in units of $e$.

$\begin{array}{crr} & (\mathrm{II}) & (\mathrm{III}) \\ \mathrm{Ru} & 0.844124 & 0.822468 \\ \mathrm{O} & -0.813500 & -0.683200 \\ \mathrm{H} & 0.503073 & 0.523061\end{array}$

Table 3: Reorganization free energies obtained from molecular dynamics simulation of the $\mathrm{Ru}(\mathrm{II})-\mathrm{Ru}(\mathrm{III})$ selfexchange reaction for different solvent models. The solute, $\left(\mathrm{Ru}(\mathrm{II})\left(\mathrm{H}_{2} \mathrm{O}\right)_{6}-\left(\mathrm{Ru}(\mathrm{III})\left(\mathrm{H}_{2} \mathrm{O}\right)_{6}\right.\right.$, is treated as nonpolarizable in all simulations. All energies are in eV.

$\begin{array}{ccccccccc} & \text { TIP3P } & \text { TIP4P } & \text { SPC } / E & \text { SPC/Fw } & \text { POL3 } & \text { AMOEBA } & \text { SWM4-NDP } & \text { Marcus } \\ \mu(\mathrm{D}) & 2.35 & 2.18 & 2.35 & 2.39 & 2.02 & 1.77 & 1.85 & \\ \epsilon_{\mathrm{s}}^{a} & 92 & 53 & 71 & 79.63 & & 81.4 & 79 & \\ \epsilon_{\mathrm{op}} & 1 & 1 & 1 & 1 & & & 1.63^{a} & \\ \lambda_{\mathrm{o}}^{b} & 0.98 \pm 0.01 & 0.88 \pm 0.02 & 1.01 \pm 0.02 & 1.16 \pm 0.04 & 0.87 \pm 0.03 & 1.03 \pm 0.03 & 0.76 \pm 0.03 & 1.42^{f} \\ \lambda_{\mathrm{o}}^{\mathrm{f} c} & 0.98 & 0.88 & 1.01 & 1.16 & 0.94 & 1.00 & 0.67 & \\ \lambda_{\mathrm{o}}^{\mathrm{i} d} & 0 & 0 & 0 & 0 & -0.07 & 0.03 & 0.09 & \\ \lambda^{\mathrm{q} e} & 1.65 & 1.57 & 1.67 & 1.77 & 1.59 & 1.74 & 1.47 & 1.93^{g}\end{array}$

${ }^{a}$ TIP3P: Ref. 42, TIP4P and SPC/E: Ref. 60, SPC/Fw: Ref. 48, AMOEBA: Ref. 35, SWM4-NDP: Ref. 43. ${ }^{b}$ Eq. 17. Statistical error $=(s / N)^{1 / 2}\left\langle\Delta E_{\mathrm{o}}\right\rangle^{1 / 2}$, where $s$ is the statistical inefficiency and $N$ the number of data points. ${ }^{61}{ }^{c}$ Eq. 18. This contribution includes the fixed quadrupole term for AMOEBA water. ${ }^{d}$ Eq. 19. ${ }^{e}$ Eq. 26 with $\lambda_{\mathrm{i}}=0.766 \mathrm{eV}$ calculated according to Eq. 21, $\Delta \lambda^{\mathrm{q}}$ taken from table 5 , and $\Delta \lambda_{\mathrm{fs}}=0.07 \mathrm{eV}$, obtained from the $1 / L$ extrapolation to infinite dilution, table $6 .{ }^{f}$ Eq. $1 . \epsilon_{\mathrm{s}}=78.3, \epsilon_{\mathrm{op}}=1.8, r_{1}=2.71 \AA, r_{2}=2.80 \AA$, and $R=5.5 \AA$. ${ }^{g}$ Eq. 26 with $\lambda_{\mathrm{i}}=0.710 \mathrm{eV}$ calculated according to Eq. $22\left(m=3 \times 10^{-26} \mathrm{~kg}, \omega_{0}=469 \mathrm{~cm}^{-1}, \tilde{\nu}_{\mathrm{O}}=532 \mathrm{~cm}^{-1},{ }^{62}\right.$ $\tilde{\nu}_{\mathrm{R}}=424 \mathrm{~cm}^{-1},{ }^{62}$ and $\left.\Delta d_{0}=0.09 \AA^{31}\right), \Delta \lambda^{\mathrm{q}}$ taken from table 5 and $\Delta \lambda_{\mathrm{fs}}=0$.

Table 4: Solvent reorganization free energies for polarizable water models at the TIP3P reference solvation structure. In model $\mathrm{X} / / \mathrm{Y}$ the solvent (or outer sphere) reorganization free energy is calculated with the potential energy function of water model $\mathrm{X}$ for configurations sampled with the potential energy function of water model $\mathrm{Y}$. The reorganization free energies are calculated according to Eqs. 17-19, $\mathrm{A}=\mathrm{Y}$. The solute is nonpolarizable in all calculations. The value for TIP3P//TIP3P is taken from table 3. All energies are in eV, and all statistical uncertainties are less than $\pm 0.02 \mathrm{eV}$.

$\begin{array}{rrrr} & \lambda_{\mathrm{o}} & \lambda_{\mathrm{o}}^{\mathrm{f}} & \lambda_{\mathrm{o}}^{\mathrm{i}} \\ \text { TIP3P//TIP3P } & 0.98 & 0.98 & 0 \\ \text { AMOEBA//TIP3P } & 0.65 & 0.79 & -0.14 \\ \text { SWM4-NDP//TIP3P } & 0.70 & 0.81 & -0.11 \\ \text { POL3//TIP3P } & 0.73 & 0.84 & -0.12 \\ \text { TIP3P//AMOEBA } & 1.25 & 1.25 & 0\end{array}$


Table 5: Quantum corrections for the exponential part and for the prefactor of the nonadiabatic rate equation 3.

$\begin{array}{ccccccccc} & \text { TIP3P } & \text { TIP4P } & \text { SPC/E } & \text { SPC/Fw } & \text { POL3 } & \text { AMOEBA } & \text { SWM4-NDP } & \text { Marcus } \\ \text { exponential } & & & & & & & & \\ \Delta \lambda_{\circ}^{\mathrm{q} a} & 0.11 & 0.10 & 0.12 & 0.17 & 0.07 & 0.08 & 0.07 & 0.13 \\ k_{\mathrm{O}}^{\mathrm{q}} / k_{\mathrm{o}}^{\mathrm{cl}} & 3.0 & 2.5 & 3.2 & 5.3 & 1.9 & 2.1 & 1.9 & 3.5 \\ \Delta \lambda^{\mathrm{q} b} & 0.18 & 0.16 & 0.19 & 0.24 & 0.13 & 0.14 & 0.13 & 0.20 \\ k^{\mathrm{q}} / k^{\mathrm{cl}} & 5.7 & 4.8 & 6.1 & 10.1 & 3.6 & 4.1 & 3.6 & 6.7 \\ \text { prefactor } & & & & & & & & \\ \Delta \lambda_{\circ}^{\mathrm{q}^{\prime}} a & 0.22 & 0.18 & 0.23 & 0.31 & 0.13 & 0.14 & 0.13 & 0.22 \\ \Delta \lambda^{\mathrm{q}^{\prime}} b & 0.35 & 0.31 & 0.36 & 0.44 & 0.26 & 0.27 & 0.26 & 0.35 \\ k^{\mathrm{q}} / k^{\mathrm{cl}} & 1.1 & 1.1 & 1.1 & 1.1 & 1.0 & 1.0 & 1.1 & 1.1 \\ \text { total } & & & & & & & & \\ k^{\mathrm{q}} / k^{\mathrm{cl}} & 6.0 & 5.2 & 6.6 & 10.9 & 3.8 & 4.2 & 3.8 & 7.7\end{array}$

${ }^{a}$ Eq. 23. Columns TIP3P to SWM4-NDP: $J_{\mathrm{o}}$ obtained from molecular dynamics simulation according to Eqs. 79. Numerical integration from 0 to $4170 \mathrm{~cm}^{-1}$, grid spacing $=1.67 \mathrm{~cm}^{-1}$. Column "Marcus": $J_{\mathrm{o}}$ calculated from experimental dielectric dispersion data ${ }^{63-65}$ according to Eq. 20. Numerical integration from 5.86 to 4167 $\mathrm{cm}^{-1}$ over all data points. ${ }^{b}$ Eq. 25 with $\Delta \lambda_{\mathrm{i}}^{\mathrm{q}}=0.066 \mathrm{eV}, \Delta \lambda_{\mathrm{i}}^{\mathrm{q}^{\prime}}=0.130 \mathrm{eV}$ calculated according to Eq. 24 with $\omega_{0}=469 \mathrm{~cm}^{-1}$ and $\lambda_{\mathrm{i}}=0.710 \mathrm{eV}$.

Table 6: Outer sphere reorganization free energy $\lambda_{\mathrm{o}}$ (Eq. 17) obtained from molecular dynamics simulation of the $\mathrm{Ru}(\mathrm{II})-\mathrm{Ru}(\mathrm{III})$ ion pair for different numbers of TIP3P water molecules per unit cell. All simulations are carried out under periodic boundary conditions. "PBC" and "Cluster" refer to the calculation of the gap energies under periodic boundary conditions and cluster conditions, respectively, carried out for the ensemble of structures obtained under periodic boundary conditions. The "Cluster" outer sphere reorganization is broken down in contributions from the 2nd and 3rd solvation shell and the remaining solvent. They are obtained by integration of $\rho_{\lambda}$ (Fig. 4) up to the distance indicated in parenthesis.

$\begin{array}{cccccc}\text { \# water/cell } & \text { PBC } & \text { Cluster }^{a} & \text { 2nd shell } & \text { 3rd shell } & \text { remaining solvent } \\ 37 & 0.957 \pm 0.026 & 0.860 \pm 0.030 & 0.86 & & \\ 63 & 0.965 \pm 0.023 & 0.822 \pm 0.040 & 0.76(5.10) & 0.06 & \\ 127 & 0.966 \pm 0.020 & 0.919 \pm 0.040 & 0.67(4.90) & 0.25 & \\ 257 & 0.970 \pm 0.022 & 1.002 \pm 0.056 & 0.67(4.91) & 0.27(7.22) & 0.06 \\ 511 & 0.979 \pm 0.014 & 0.964 \pm 0.032 & 0.62(4.88) & 0.26(7.25) & 0.08 \\ 1022 & 1.058 \pm 0.033 & 1.089 \pm 0.039 & 0.61(4.87) & 0.32(6.96) & 0.16 \\ 10005 & 1.037 \pm 0.024 & 1.036 \pm 0.038 & 0.60(4.84) & 0.29(6.99) & 0.15 \\ \infty^{b} & 1.052 \pm 0.023 & 1.120 \pm 0.042 & & & \\ \infty^{c} & 1.023 \pm 0.018 & 1.052 \pm 0.029 & & & \end{array}$

${ }^{a}$ Center of mass of the two Ru ions was translated to the center of the box and all of the water molecules wrapped into the central box. ${ }^{b} 1 / L$ extrapolation, Figure 3 A. ${ }^{c} 1 / L^{2}$ extrapolation, Figure 3 B. 
Figure 1: Radial distribution function between $\mathrm{Ru}$ ions and oxygen atoms of solvent water molecules obtained from molecular dynamics simulation of a solution of the $\mathrm{Ru}(\mathrm{II})-\mathrm{Ru}(\mathrm{III})$ ion pair and 511 water molecules. The $\mathrm{Ru}(\mathrm{II})-\mathrm{Ru}(\mathrm{III})$ distance is fixed at $5.5 \AA$. Data were sampled over 1 ns and collected in bins of with $0.01 \AA$. The distributions were smoothed by convolution with a Gaussian of width $0.03 \AA$.

Figure 2: Outer sphere quantum correction of the exponential part of the nonadiabatic rate equation 3 for the $\mathrm{Ru}(\mathrm{II})-\mathrm{Ru}(\mathrm{III})$ electron self-exchange reaction. The outer sphere spectral density function $J_{\mathrm{o}}$ is obtained from molecular dynamics simulation and calculated according to Eqs. 7-9 for the following water models: Panel A: TIP3P $(\cdots \cdots)$, TIP4P $(---)$, SPC/E $(--)$, SPC/Fw $(-\cdot-\cdot)$; Panel B: POL3 $(-\cdot-)$, AMOEBA $(-\cdots-)$, SWM4-NDP $(\cdot--\cdot) ; y=\beta \hbar \omega / 4$. The spectra were smoothed by convolution with a Gaussian of width $30 \mathrm{~cm}^{-1}$. The estimate based on experimental dispersion data (Eq. 20) is drawn in solid lines (_- ) in panel A and B. The frequency-resolved quantum correction of the outer sphere reorganization free energy, $\Delta \lambda_{o}^{q}(\omega)$, is shown in panel C. It was obained according to Eq. 23 by numerical evaluation of the integrals Eqs. 4 and 6 between 0 and $\omega$.

Figure 3: Outer sphere reorganization free energy as a function of box length, $1 / L$ (panel A) and $1 / L^{2}$ (panel B). $L=V^{1 / 3}$, where $V$ is the volume of the simulation box. The seven systems contain $37,63,127,257,511,1022$, and 10005 water molecules, respectively. The gap energies Eq. 17 are calculated under periodic boundary conditions (PBC) and under cluster conditions. Best linear fits of the data are shown in dashed lines. See section 4.4 and table 6 for further details.

Figure 4: Outer sphere radial reorganization free energy density $\rho_{\lambda}$, Eq. 30, for the $\mathrm{Ru}(\mathrm{II})-\mathrm{Ru}(\mathrm{III})$ self-exchange reaction. The systems contain a variable number of TIP3P water molecules: $37(\cdots \cdots), 63(-\cdot-\cdot), 511(-\cdots-)$, $1022(---), 10005(-)$. The distance $d$ is defined as the shortest of the two distances between a water molecule and the two Ru-ions. The data are collected in bins of width $0.01 \AA$, and smoothed by convolution with a Gaussian of width $0.03 \AA$. The reorganization density integrated up to a distance $d$ is shown in the inset. The distance between the center of mass of the solute and the closest box edge is indicated by a vertical dash for the three largest simulation systems. See section 4.4 and table 6 for further details. 


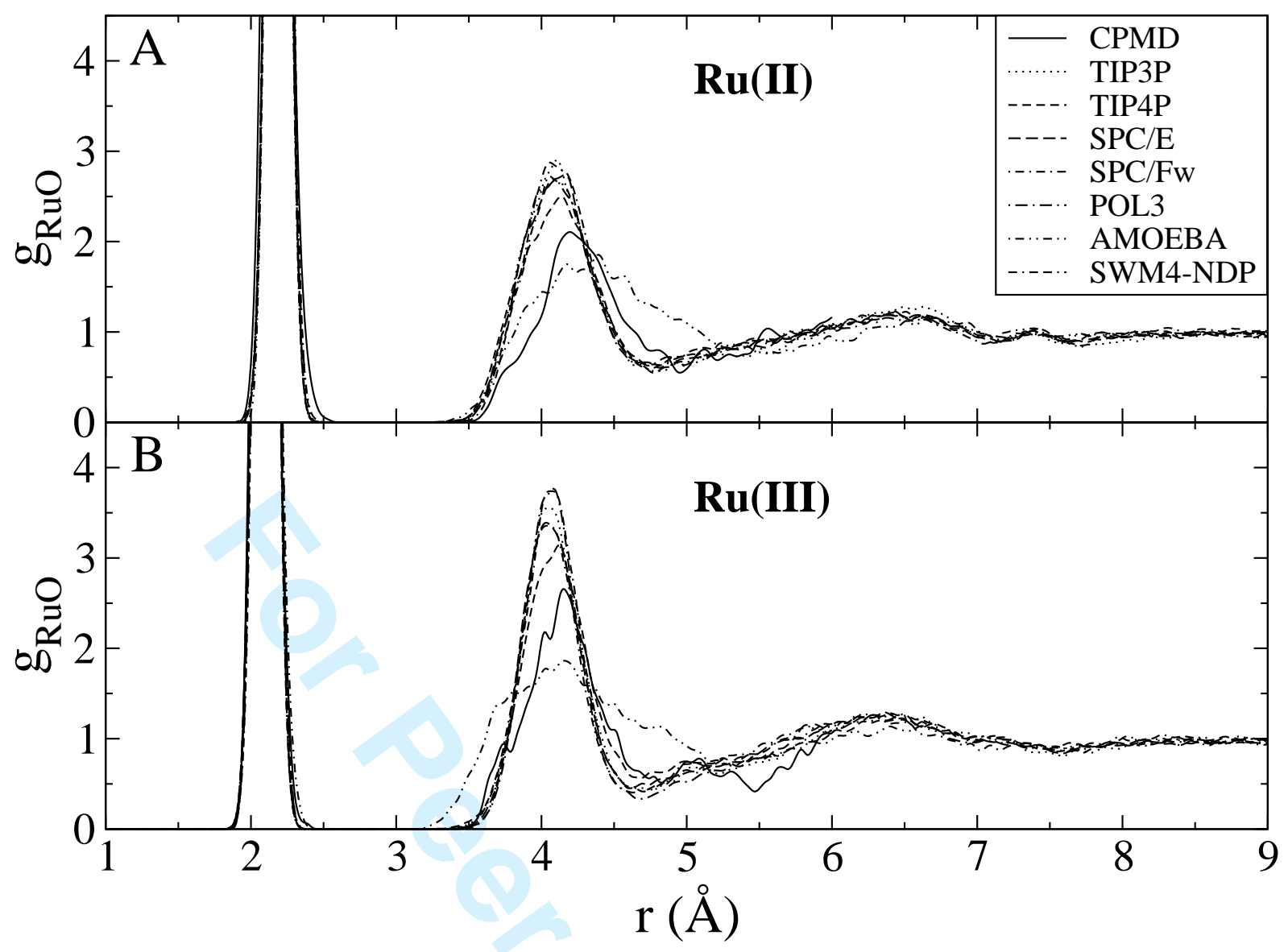

Figure 1. 


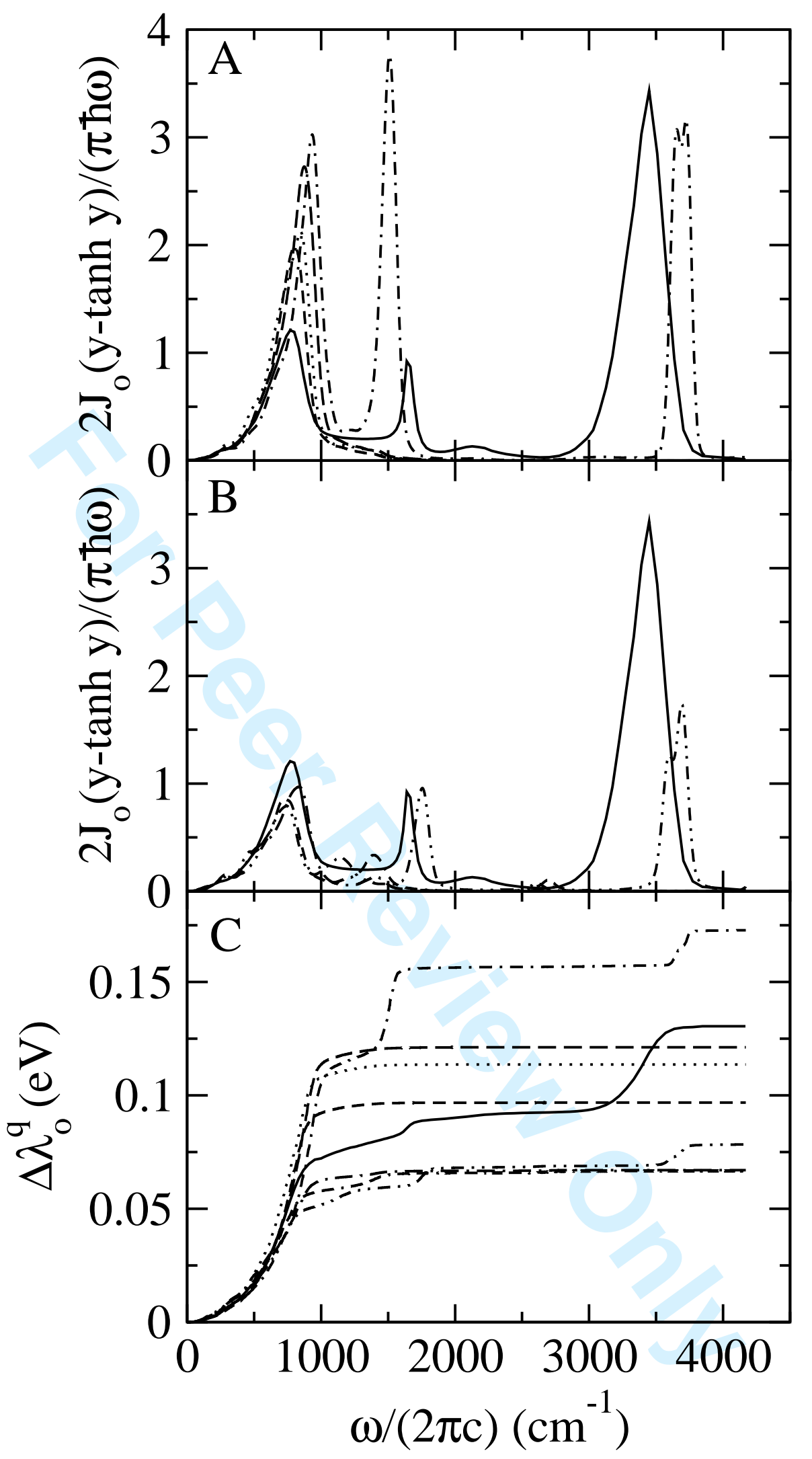

Figure 2. 

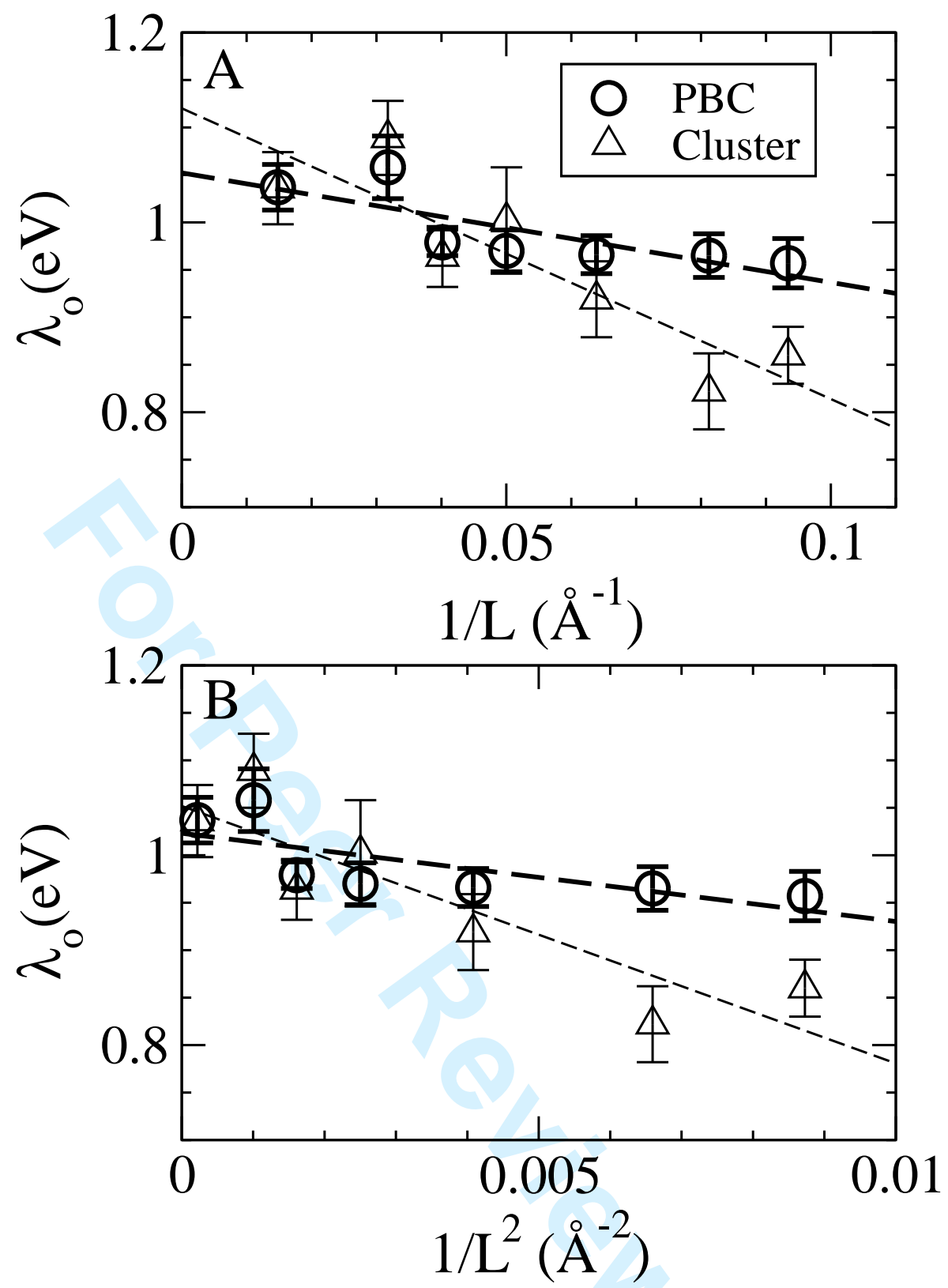

Figure 3 . 


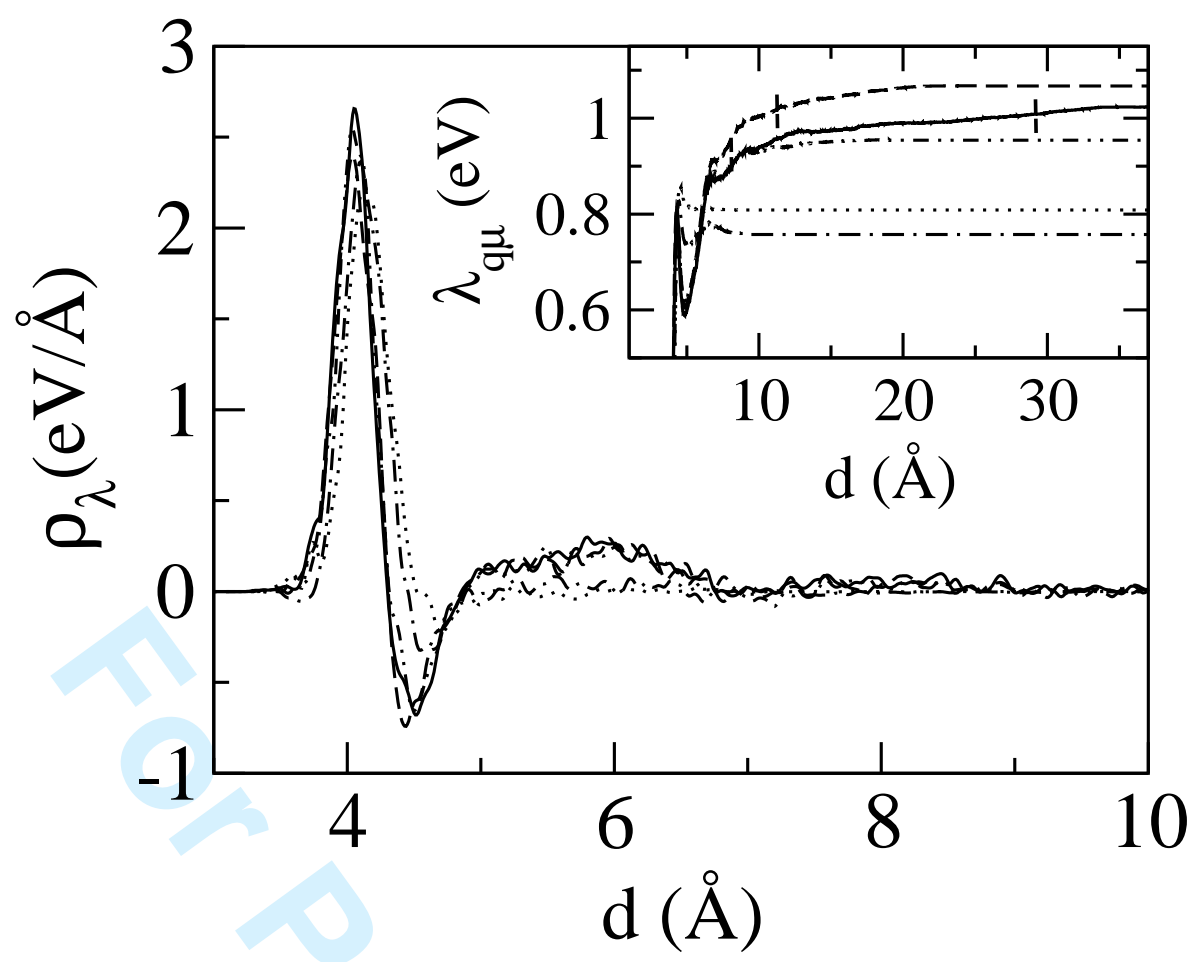

1
2
3
4
5
6
7
8
9
10
11
12
13
14
15
16
17
18
19

Figure 4 . 


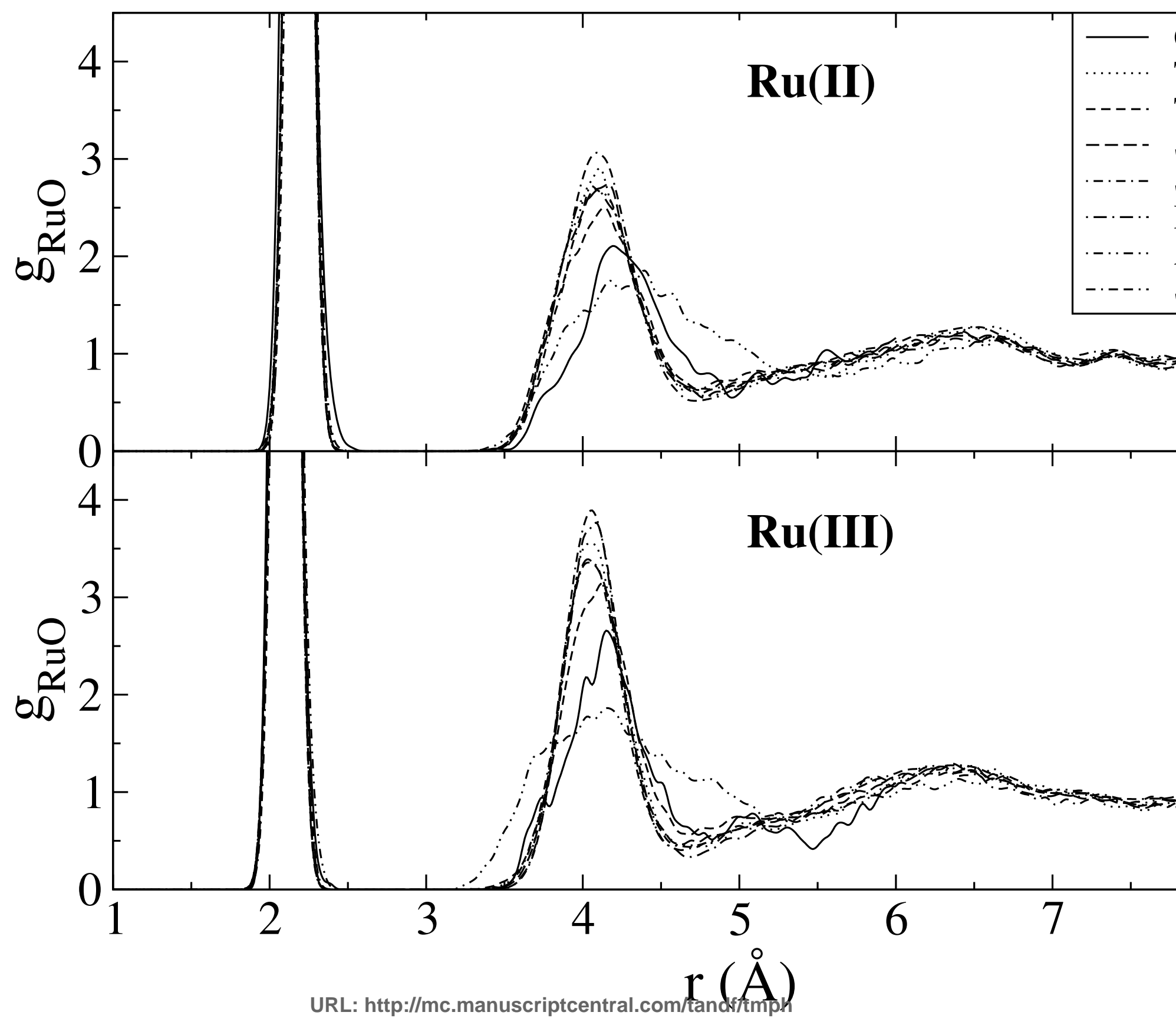




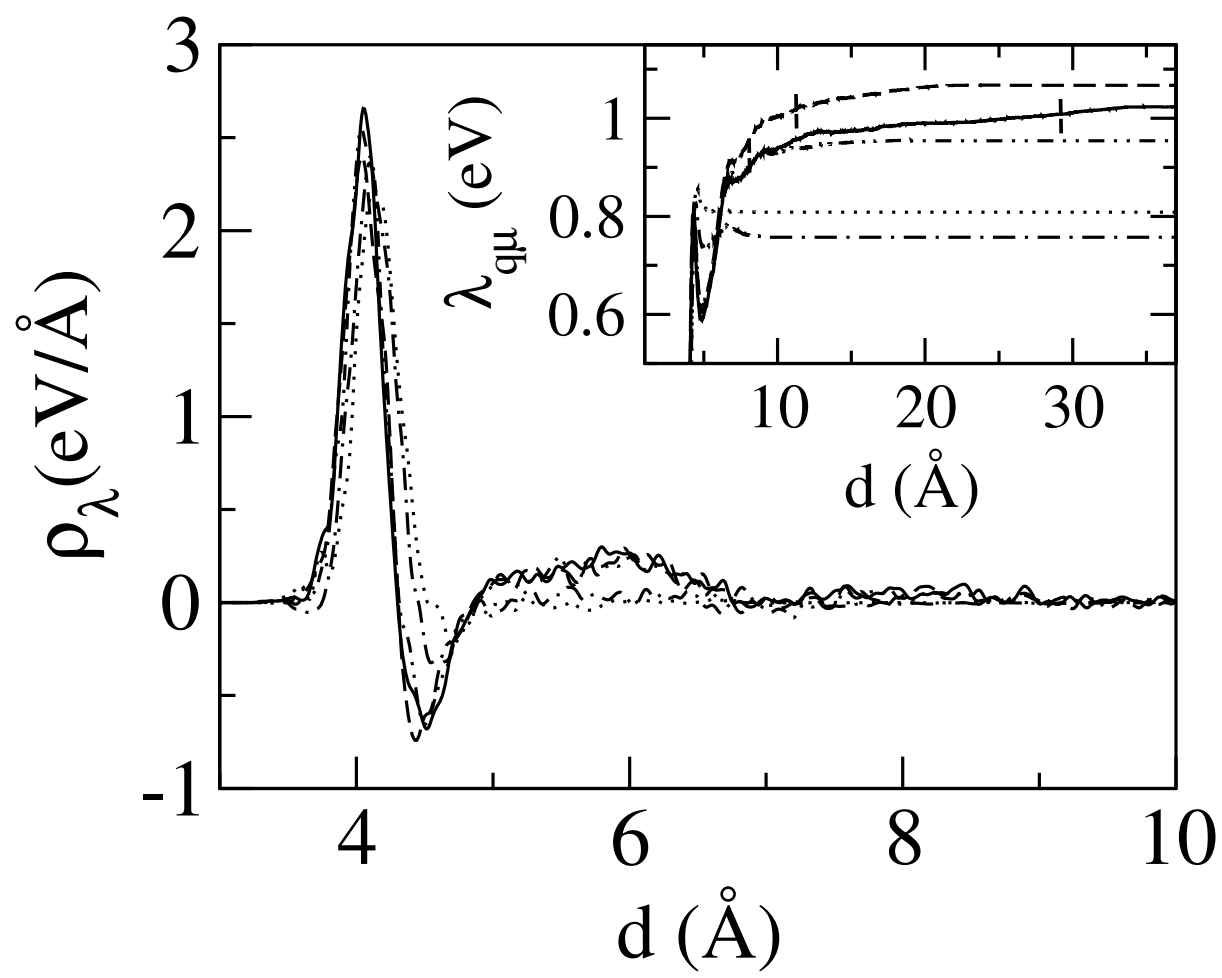

URL: http://mc.manuscriptcentral.com/tandf/tmph 
1
2
3
4
5
6
7
8
9

10

11

12

13

14

15

16

17

18

19

20

21

22

23

24

25

26

27

28

2

3

31

32

33

34

35

36

37

38

39

40

41

42

43

44

45

46

47

48

49

50

51

52

53

54

55

56

57

58

59

60

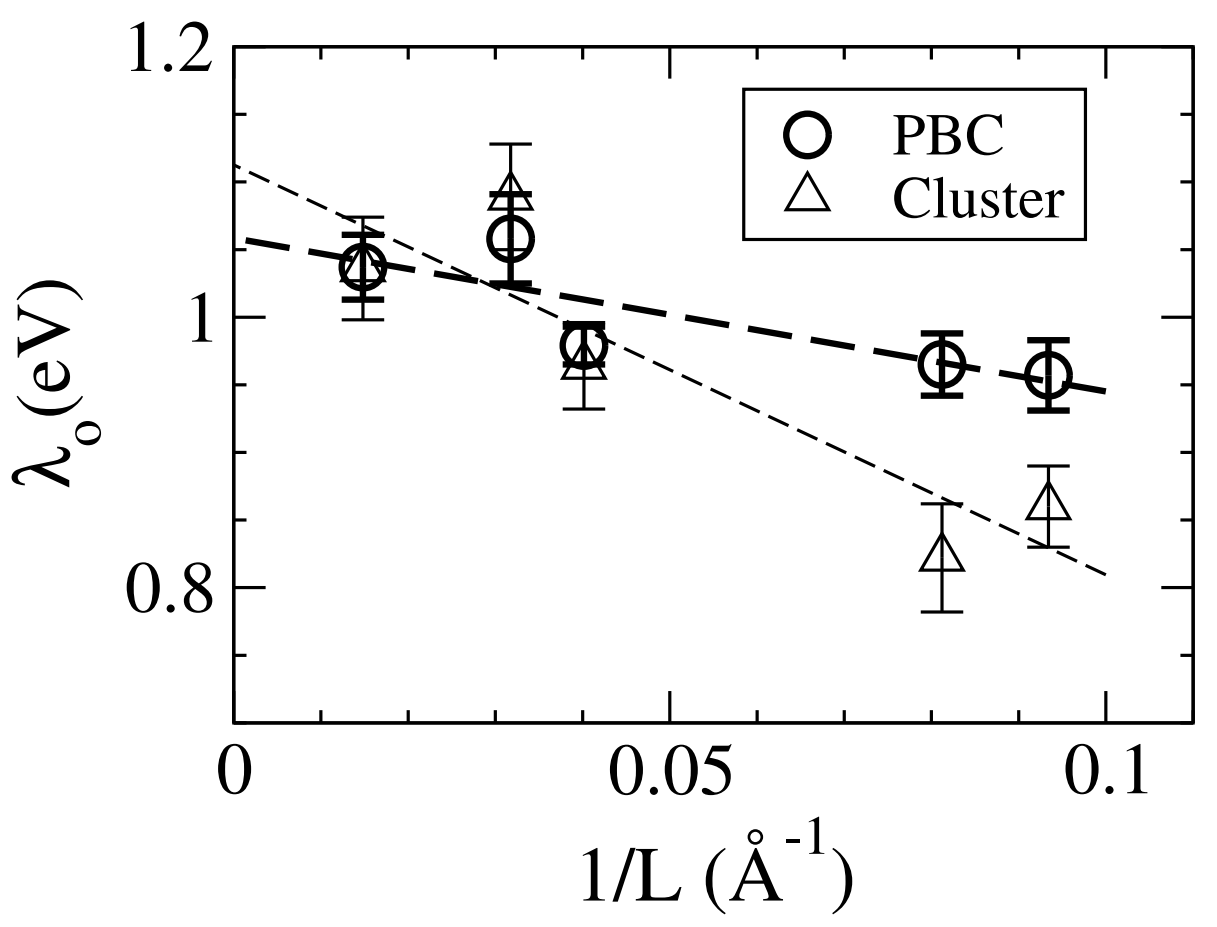

URL: http://mc.manuscriptcentral.com/tandf/tmph 